\title{
Risk-sensitive Consumption and Savings under Rational Inattention*
}

\author{
Yulei Luo ${ }^{\dagger}$ \\ School of Economics and Finance \\ University of Hong Kong
}

\author{
Eric R. Young $\ddagger$ \\ Department of Economics \\ University of Virginia
}

January 18,2008

\begin{abstract}
This paper studies the consumption-savings behavior of households who have risk-sensitive preferences and suffer from limited information-processing capacity (rational inattention or RI). We find that the model displays a wide range of observational equivalence properties, implying that consumption and savings data cannot distinguish between models with risksensitivity, robustness, rational inattention, or rational expectations, in any combination. We then show that the welfare costs from RI are much larger for risk-sensitive households than any other observationally-equivalent settings. Thus, a potential method for testing risk-sensitivity vs. robustness is to ask agents how much they would pay to receive better information about the state of the world.
\end{abstract}

JEL Classification Numbers: C61, D81, E21.

Keywords: Rational Inattention, Consumption, Precautionary Savings, Risk-sensitive Control, Robust Control.

${ }^{*}$ We thank Chris Sims and Chi-Wa Yuen for helpful discussion, as well as seminar participants at the 2007 North American Summer Meetings of the Econometric Society, University of Hong Kong, and Chinese University of Hong Kong. Luo thanks the Competitive Earmarked Research Grant (CERG) in Hong Kong for financial support. Young thanks the Bankard Fund for Political Economy at Virginia for financial support. All remaining errors are our responsibility.

$\dagger$ Corresponding author. School of Economics and Finance, University of Hong Kong, Hong Kong, yluo@econ.hku.hk.

$\ddagger$ Department of Economics, University of Virginia, Charlottesville, VA 22904, ey2d@virginia.edu. 


\section{Introduction}

Sims (1998) first argued that rational inattention (RI) is a plausible method for introducing sluggishness, randomness, and delay into economic models. In his formulation agents have finite Shannon channel capacity, limiting their ability to process signals about the true state of the world. As a result, an impulse to the economy induces only gradual responses by individuals, as their limited capacity requires many periods to discover just how much the state has moved. Sims (2003) and Luo (2007) use this model to explore anomalies in the consumption literature, particularly the well-known 'excess sensitivity' and 'excess smoothness' puzzles, employing a linear-quadratic version of the standard permanent income model (as in Hall 1978). In that model RI is equivalent to confronting the household with a signal extraction problem regarding the value of permanent income (as in Friedman 1957) but permitting the agents to choose the distribution of the noise terms, subject to their limited capacity. ${ }^{1}$ With normal innovations to income the optimal distribution of noise is also normal, leading to a simple and analytically tractable problem.

Of course, the linear-quadratic model has some undesirable features as well. One objection that is particularly relevant for this paper is that it satisfies the certainty equivalence property, ruling out any response of saving to uncertainty (that is, precautionary behavior). ${ }^{2}$ Given that the important component of RI is the introduction of endogenous uncertainty into the household problem, it is not particularly desirable to use a model in which this uncertainty cannot manifest itself in decision rules. Fully nonlinear versions of the RI problem are solved in Sims $(2005,2006)$, Lewis (2006), Batchuluun, Luo, and Young (2007), and Tutino (2007); these papers show that the precautionary aspect of RI is important when channel capacity is small (which it must be to produce any interesting results). But the models solved in those papers have either very short horizons or extremely simple setups due to numerical obstacles - the state of the world is the distribution of perceived states and this distribution is not well-behaved (it is not generally a member of a known class of distributions and tends to have 'holes,' making it difficult to characterize with a small number of parameters). It is important to find a class of models that can produce precautionary behavior while maintaining tractability in the RI setup, if the properties of finite channel capacity are going to be thoroughly explored.

One class of models that satisfies these desiderata is the risk-sensitive permanent income model from Hansen, Sargent, and Tallarini (1999). ${ }^{3}$ In this model agents effectively compute expectations through a distorted lens, increasing their effective risk aversion by overweighting

\footnotetext{
${ }^{1}$ This point is made explicitly in Luo and Young (2006) in the context of the stochastic growth model.

${ }^{2}$ See Simon (1956) and Theil (1957).

${ }^{3}$ Possibly the first contribution to this literature is van der Ploeg (1993). Hansen and Sargent (2004) demonstrate that the risk-sensitive model displays a form of certainty equivalence, but that it generates precautionary savings anyway.
} 
negative outcomes. The resulting decision rules depend explicitly on the variance of the shocks, producing precautionary savings, but the value functions are still quadratic functions of the states, leading again to the optimality of Gaussian noise in the RI model. Thus, we preserve the tractability of the LQ PIH model without being forced to accept certainty equivalence; for future reference we denote the model with risk sensitivity and rational inattention as the RSRI model. ${ }^{4}$ Formally, one can view risk-sensitive agents as ones who have non-state-separable preferences, as in Epstein and Zin (1989), but with a restricted value for the intertemporal elasticity of substitution (see Tallarini 2000).

A second class of models that produces precautionary savings but remains within the class of LQ-Gaussian models is the robust control model of Hansen and Sargent (1995). In robust control problems, agents are concerned about the possibility that their model is misspecified in a manner that is difficult to detect statistically; as a result, they choose their decisions as if the subjective distribution over shocks was chosen by a malevolent nature in order to minimize their expected utility (that is, the solution to a robust decision-maker's problem is the equilibrium of a max-min game between the decision-maker and nature). Hansen and Sargent (2007) present an observational equivalence result for RS and Robust models for consumption and savings decisions; any consumption path that could be generated by a model featuring risk sensitivity can also be generated by a model with robustness. Thus, introducing RI into the Robust model - which we denote the RB-RI model - is again straightforward, since the model retains the optimality of Gaussian noise.

Our purpose in this paper is to study the properties of RS-RI and RB-RI models. In particular, we are interested in two main questions. The first question involves the question of observational equivalence - do RS-RI and RB-RI models possess combinations of parameters such that their implied consumption-savings rules are the same? And can those decision rules also be generated by a standard RE and standard RI model? To preview the results, which we discuss in more detail below, the answer is yes - any member of the class of LQ-Gaussian models can produce the same consumption function with appropriate adjustments in some combination of the discount factor, the capacity constraint on information processing, the degree of risk sensitivity, or the preference for robustness. The second question is how the costs of finite information-processing capacity differ within the class of observationally-equivalent models; that is, do observationally-equivalent agents suffer more or less from RI depending on which model generates their behavior? As a preview, we find here that the costs are not equal - RS-RI models produce larger welfare losses from RI than do RB-RI and RI models. Thus, we identify a potential channel through which risk sensitivity and robustness can be disentangled - simply

\footnotetext{
${ }^{4}$ Of course, quadratic utility carries with it other objectionable properties, such as increasing relative risk aversion.
} 
ask agents what they would pay to relax their Shannon channel constraint.

Our first step in studying the various RI models is to perform a reduction of the state space. Multivariate versions of the RI model require a constraint we term 'no subsidization.' With more than one state variable, agents can allocate their attention differently across these variables and thus reduce their uncertainty at different rates; RI requires that the uncertainty regarding one variable cannot be increased in order to reduce uncertainty regarding another, as this would permit reductions that exceed the channel capacity. This constraint is nonlinear when the dimension is larger than one, even in the LQ model, so multivariate versions of the RI model are not any more tractable than the nonlinear models mentioned above. We show that a PIH model with a general labor income process can be reduced to a model with a unique state variable - permanent income - that has iid innovations, enabling us to study a model with an empirically-reasonable income process within the LQ setup.

We then present the basic risk-sensitive (RS) model and derive some results regarding the behavior of consumption. We show explicitly that precautionary savings is increasing in the parameter that measures risk sensitivity and that the marginal propensity to consume out of permanent income is decreasing. From here we proceed by introducing finite Shannon channel capacity; RI implies that behavior is as if the agent were observing a noisy signal of next period's true permanent income, so the agent uses the Kalman filter to update the perceived state. Since the evolution of the true state is affected by precautionary motives, the evolution of the perceived state is as well. Thus, RS and RI interact - while the household uses the same Kalman gain parameter independent of the degree of risk sensitivity, the mean value of the signal received increases with RS so the perceived state evolves differently.

Because we are able to provide closed-form solutions to the RS-RI model we can provide two general results. First, introducing RI into the RS model improves the predictions about the aggregate joint dynamics of consumption and income; this result is obtained because the basic RS model actually reduces the ability of the model to match the sensitivity of consumption to income changes. ${ }^{5}$ Second, we show that observational equivalence holds for quantities - we can find more than one combination of discount factors and risk-sensitivity that imply the same savings and consumption behavior, whether RI is present or not. The intuition is the same as that in Hansen, Sargent, and Tallarini (1999) - an agent with RS looks just like a standard but more patient agent. This equivalence extends to the RI models as well, complementing the result in Luo (2007) that RE and RI models are also observationally equivalent.

We then turn to the RB-RI model. Here again we derive closed-form solutions and generate an observational equivalence result, which now extends in multiple directions. That is, we show

\footnotetext{
${ }^{5}$ This fact explains the use of habit formation and preference shocks in RS models (such as Hansen, Sargent, and Tallarini 1999).
} 
that the RB-RI model produces the same consumption-savings decisions as the RS-RI model and, therefore, the RE and RI models. The intuition remains the same as above - with a preference for robust decision-making the agent chooses to save more than before, appearing more patient when viewed through the lens of a standard RE or RI model. Econometrically, these observational equivalence properties imply that it is impossible to test for the presence of RI, RS, or robustness using this model and consumption-savings data alone.

We then ask whether anything fundamentally distinguishes the RS-RI model from the RI and RB-RI models. We find a key difference when we measure the welfare costs of RI - that is, how much would an agent pay to increase their information-processing capacity to $\infty$ ? Luo (2007) studied the costs of RI in the standard model and concluded that they are trivial. Generalizing his result to the RS-RI and RB-RI models, we find that the costs may not be trivial; in particular, agents in the RS-RI model can suffer very large losses from RI. In fact, we can prove that the RS-RI model implies that the welfare losses from RI are necessarily larger than those in RB$\mathrm{RI}$, and that this gap is increasing as channel capacity converges to 0 . One key difference is that agents in the RI-RS model change their consumption behavior as their capacity changes, whereas RB-RI agents do not.

The remainder of the paper is organized as follows. Section 2 presents two PIH models with general income processes and discusses some results regarding optimal consumption-savings decisions and the joint dynamics of aggregate consumption and income (these models are the standard PIH model and the RS model). Because the RB model is equivalent to the RS model, we do not explicitly present and discuss it here, preferring to defer it to the following section. Section 3 introduces RI into both the RS and RB models and the behavior of consumption and savings; we provide closed-form solutions for all models here. Section 4 presents our battery of observational equivalence results. Section 5 then discusses the welfare costs of RI. Section 6 concludes with a discussion of some potential directions that the literature could proceed.

\section{Two Rational Expectations Permanent Income Models}

In this section, we present two benchmark models. In the first model, we show that within the LQG framework, the multivariate permanent income model with general income processes can be reduced to the univariate model with iid innovations to permanent income that can be solved in closed-form solution. We then present the second model in which risk-sensitivity is introduced into the univariate PIH model in Section 2.1.

\subsection{The Standard Permanent Income Model}

We first present a standard rational expectation (RE) version of the PIH model with a general income process. We then show that it can be reduced to a univariate PIH model with iid 
innovations to permanent income. Households solve the dynamic consumption-savings problem

$$
\max _{\left\{c_{t}\right\}_{t=0}^{\infty}} E_{0}\left[\sum_{t=0}^{\infty} \beta^{t} u\left(c_{t}\right)\right]
$$

subject to

$$
w_{t+1}=R\left(w_{t}-c_{t}\right)+y_{t+1},
$$

where $u\left(c_{t}\right)=-\frac{1}{2}\left(c_{t}-\bar{c}\right)^{2}$ is the period utility function, $\bar{c}>0$ is the bliss point, equation (2.2) is the flow budget constraint, $c_{t}$ is consumption, $w_{t}$ is cash-on-hand (or market resources), $y_{t}$ is a general income process with Gaussian white noise innovations, $\beta$ is the discount factor, $R$ is the constant gross interest rate at which the consumer can borrow and lend freely. ${ }^{6}$ For the rest of the paper we will restrict attention to points where $c_{t}<\bar{c}$, so that utility is increasing and concave. This specification follows that in Hall (1978) and Flavin (1981) and implies that optimal consumption is determined by permanent income:

$$
c_{t}=\left(1-\frac{1}{\beta R^{2}}\right) s_{t}-\frac{1}{R-1}\left(1-\frac{1}{\beta R}\right) \bar{c}
$$

where

$$
s_{t}=w_{t}+\sum_{j=1}^{\infty} R^{-j} E_{t}\left[y_{t+j}\right]
$$

is the expected present value of lifetime resources, consisting of financial wealth plus human wealth. If $s_{t}$ is defined as a new state variable, we can reformulate the above PIH model as

$$
v\left(s_{0}\right)=\max _{\left\{c_{t}\right\}} E_{0}\left[\sum_{t=0}^{\infty} \beta^{t} u\left(c_{t}\right)\right]
$$

subject to

$$
s_{t+1}=R\left(s_{t}-c_{t}\right)+\zeta_{t+1}
$$

a formal proof is contained in Luo (2007). The stochastic term $\zeta_{t+1}$ is equal to

$$
\zeta_{t+1}=\sum_{j=t+1}^{\infty} R^{-j+(t+1)}\left(E_{t+1}-E_{t}\right)\left[y_{j}\right]
$$

\footnotetext{
${ }^{6}$ As in Hansen and Sargent (2007) or Hansen, Sargent, and Tallarini (1999), we can also interpret this problem as applying to a linear production technology that generates consumption and capital,

$$
c_{t}+k_{t}=R_{k} k_{t-1}+y_{t}
$$

where $R_{k}$ is the net physical return on capital and investment is given by $i_{t}=k_{t}-k_{t-1}$. If the allocation is decentralized the return coincides with the risk-free interest rate $R$.
} 
that is, $\zeta_{t+1}$ the time $(t+1)$ innovation to permanent income. $v\left(s_{0}\right)$ is the consumer's value function under RE. By defining a new state variable $s_{t}$, the original multivariate optimization problem has been reduced to a univariate problem; under quadratic utility this model leads to the well-known random walk result of Hall (1978):

$$
\Delta c_{t+1}=-\frac{\beta R-1}{\beta R}\left(c_{t}-\bar{c}\right)+\frac{\beta R^{2}-1}{\beta R^{2}} \zeta_{t+1}
$$

if $\beta R=1$ the change in consumption depends neither on the past history of labor income nor on anticipated changes in labor income. We also point out the well-known result that the standard PIH model with quadratic utility implies the certainty equivalence property holds; thus, uncertainty has no impact on optimal consumption, so that there is no precautionary saving in the sense of Kimball (1990). We assume for the remainder of this section that $\beta R=1$, since this setting is the only one that implies stationary consumption.

Let $k_{t}=w_{t}-c_{t}$ denote end of period financial wealth. Then $i_{t}=k_{t}-k_{t-1}$ is a measure of saving (or investment). Substituting this expression into (2.2) and using (2.6) and (2.8) we obtain $\Delta k_{t+1}=0$; thus, there is no wealth accumulation in response to changes in permanent income, as all such changes are allocated to consumption.

For simplicity, following Pischke (1995) we suppose that income $y_{t}$ can be expressed as the sum of aggregate permanent and idiosyncratic transitory components:

$$
y_{t+1}=y_{t+1}^{p}+y_{t+1}^{t}
$$

where the superscripts $p$ and $t$ denote permanent and transitory, respectively. Each of these components follows its own stochastic process; $y_{t+1}^{p}$ follows a random walk

$$
y_{t+1}^{p}=y_{t}^{p}+\varepsilon_{t+1}
$$

and $y_{t+1}^{t}$ follows an iid process

$$
y_{t+1}^{t}=\bar{y}+\epsilon_{t+1},
$$

where $\varepsilon_{t+1}$ and $\epsilon_{t+1}$ are white noises with mean 0 and variance $\omega_{\varepsilon}^{2}$ and $\omega_{\epsilon}^{2}$ respectively. Substituting these income processes into (2.8) gives

$$
\Delta c_{t+1}=\varepsilon_{t+1}+\frac{R-1}{R} \epsilon_{t+1} .
$$

The different load factors on the two shocks reflects the standard consumption-smoothing motives: consume all of a permanent increase in income $\left(\varepsilon_{t+1}\right)$ but only the annuity value of a transitory one $\left(\epsilon_{t+1}\right)$. 
We now want to derive some properties of aggregate consumption. Suppose that there are a continuum of consumers in the model economy. Given the expression of the change in individual consumption, (2.12), aggregating across all consumers yields the change in aggregate consumption as

$$
\Delta C_{t+1}=\varepsilon_{t+1}
$$

so that aggregate consumption $C_{t+1}$ is unpredictable using past information and the smoothness ratio of aggregate consumption to income is $1 .^{7}$ In other words, the predictions of the standard $\mathrm{PIH}$ model for the joint behavior of aggregate consumption and income is not consistent with the empirical evidence; in the US data aggregate consumption growth is much smoother than income and is sensitive to past information. ${ }^{8}$ These two anomalies have been termed the excess smoothness and excess sensitivity puzzles in the literature. ${ }^{9}$ Furthermore, as documented in Reis (2006), the impulse response of aggregate consumption to aggregate income takes a humpshaped form, which means that aggregate consumption reacts to income shocks gradually. The standard RE PIH model cannot capture this feature in the US data.

\subsection{The Risk-sensitive Permanent Income Model}

Risk-sensitivity (RS) was first introduced into the LQ-Gaussian framework by Jacobson (1973) and extended by Whittle (1981,1990). Exploiting the recursive utility framework of Epstein and Zin (1989), Hansen and Sargent (1995) introduce discounting into the RS specification and show that the resulting decision rules are time-invariant. Hansen, Sargent, and Tallarini (1999) use this model to study a risk-sensitive version of the PIH model and show how RS alters the choices of consumption, investment, and asset prices; Tallarini (2000) addresses many of the same issues within the stochastic growth model.

We use an RE version of risk-sensitive control based on Kreps and Porteus (1978) preferences with an exponential certainty equivalence function. The household problem can be written recursively as

$$
v\left(s_{t}\right)=\max _{c_{t}}\left\{-\frac{1}{2}\left(c_{t}-\bar{c}\right)^{2}+\beta \mathcal{R}_{t}\left[v\left(s_{t+1}\right)\right]\right\},
$$

subject to (2.6). The distorted expectation operator $\mathcal{R}_{t}$ is defined by

$$
\mathcal{R}_{t}\left[v\left(s_{t+1}\right)\right]=-\frac{1}{\alpha} \log E_{t}\left[\exp \left(-\alpha v\left(s_{t+1}\right)\right)\right]
$$

where $\alpha>0$ measures higher risk aversion vis a vis the von Neumann-Morgenstern specification. Solving this optimization problem yields the following proposition.

\footnotetext{
${ }^{7}$ The idiosyncratic shock, $\epsilon$, would be cancelled out after aggregating over all consumers.

${ }^{8}$ In the US data, the smoothness ratio is around 0.5.

${ }^{9}$ See Deaton (1992) for a recent review.
} 
Proposition 1. The value function for the $R S$ model is

$$
\begin{aligned}
v\left(s_{t}\right) & =-\frac{1}{2} \frac{\beta R^{2}-1}{\beta R^{2}-\alpha \omega_{\zeta}^{2}} s_{t}^{2}+\frac{R\left(\beta R^{2}-1\right)}{\left(\beta R^{2}-\alpha \omega_{\zeta}^{2}\right)(R-1)} \bar{c} s_{t}- \\
& \left(\frac{1}{2} \frac{R^{2}\left(\beta R^{2}-1\right)}{(R-1)^{2}\left(\beta R^{2}-\alpha \omega_{\zeta}^{2}\right)} \bar{c}^{2}-\frac{\beta}{1-\beta} \frac{1}{2 \alpha} \log \left(1-2 \alpha A \omega_{\zeta}^{2}\right)\right),
\end{aligned}
$$

the consumption function is

$$
c_{t}=\frac{\beta R^{2}-1}{\beta R^{2}-\alpha \omega_{\zeta}^{2}} s_{t}+\frac{\left(R-\beta R^{2}+(1-R) \alpha \omega_{\zeta}^{2}\right) \bar{c}}{\left(\beta R^{2}-\alpha \omega_{\zeta}^{2}\right)(R-1)},
$$

and permanent income evolves according to

$$
s_{t+1}=R \frac{1-\alpha \omega_{\zeta}^{2}}{\beta R^{2}-\alpha \omega_{\zeta}^{2}} s_{t}-R \frac{\left(R-\beta R^{2}+(1-R) \alpha \omega_{\zeta}^{2}\right) \bar{c}}{\left(\beta R^{2}-\alpha \omega_{\zeta}^{2}\right)(R-1)}+\zeta_{t+1} .
$$

Proof. See Appendix 7.2.

Equation (2.17) shows that the parameter controlling risk-sensitivity, $\alpha$, affects both the marginal propensity to consume (MPC) out of perceived permanent income, $\frac{\beta R^{2}-1}{\beta R^{2}-\alpha \omega_{\zeta}^{2}}$, and the precautionary savings increment, $\frac{\alpha \omega_{\zeta}^{2}}{R-\alpha \omega_{\zeta}^{2}} \bar{c} .{ }^{10}$ The larger the value of $\alpha$ the larger the MPC and the larger the precautionary saving increment (we impose the restriction $\beta R^{2}>1>\alpha \omega_{\zeta}^{2}$ so that precautionary savings is positive), since

$$
\begin{array}{r}
\frac{\partial\left(\frac{\beta R^{2}-1}{\beta R^{2}-\alpha \omega_{z}^{2}}\right)}{\partial \alpha}>0 \\
\frac{\partial\left(\frac{\left(R-\beta R^{2}+(1-R) \alpha \omega_{\zeta}^{2}\right)}{\left(\beta R^{2}-\alpha \omega_{\zeta}^{2}\right)(R-1)}\right)}{\partial \alpha}>0 .
\end{array}
$$

As its name suggests, risk sensitivity makes agents more concerned about outcomes that are unfavorable; one way to minimize the probability of bad outcomes (here, outcomes where the marginal utility of consumption becomes very large) is to save a bit more, reducing consumption relative to the certainty equivalent setup where $\alpha=0$. In the long run agents will have more wealth as the extra precautionary savings generates more returns.

\footnotetext{
${ }^{10}$ Given the definition of $s_{t}$ in (2.6), the MPC out of permanent income equals the MPC out of financial wealth $w_{t}$ and human wealth $\sum_{j=1}^{\infty} R^{-j} E_{t}\left[y_{t+j}\right]$.
} 
Relative risk aversion over wealth gambles is given by

$$
r\left(s_{t}\right)=\frac{1}{\frac{R}{R-1} \frac{\bar{c}}{s_{t}}-1}
$$

which is an decreasing function of $\frac{\bar{c}}{s_{t}}$. Thus, $\alpha$ does not directly impact risk aversion in this model; what matters for risk aversion is only how close current permanent income is to the bliss point. $\alpha$ increases $s_{t}$, however, and thus indirectly increases the risk aversion of the agent; the IRRA property of quadratic utility functions remains in the RS setting.

We now present some basic results from the RS model, mainly to facilitate comparisons to the RI model of the next section. The change in consumption can be written as

$$
\Delta c_{t+1}=-\frac{\beta R^{2}-R-(1-R) \alpha \omega_{\zeta}^{2}}{\beta R^{2}-\alpha \omega_{\zeta}^{2}}\left(c_{t}-\bar{c}\right)+\zeta_{t+1} .
$$

Equation (2.19) has two important implications for our purposes. Setting $\alpha>0$ implies that consumption growth becomes more volatile relative to income and becomes more sensitive to unanticipated changes in permanent income, since $\frac{\beta R^{2}-1}{\beta R^{2}}<1$. Furthermore, consumption growth is not mean zero unless $c_{t}=\bar{c}$ even when $\beta R=1$; indeed, when $\beta R=1$ consumption will drift upward over time. Counteracting this drift requires $\widehat{\beta} R<1$ such that the tendency to save for precautionary reasons is exactly counterbalanced by the impatience effect of increasing consumption; this result is the basis of the observational equivalence result in Hansen, Sargent, and Tallarini (1999).

As in the last subsection, substituting the specified income processes, (2.9), (2.10), and (2.11), into (2.19) gives

$$
\Delta c_{t+1}=\frac{\beta R^{2}-1}{\beta R^{2}-\alpha \omega_{\zeta}^{2}}\left[\left(\frac{R}{R-1} \varepsilon_{t+1}+\epsilon_{t+1}\right)+\left(\rho_{1}-1\right) \frac{\frac{R}{R-1} \varepsilon_{t}+\epsilon_{t}}{1-\rho_{1} L}\right],
$$

where $\rho_{1}=R \frac{1-\alpha \omega_{\zeta}^{2}}{\beta R^{2}-\alpha \omega_{\zeta}^{2}} \in(0,1)$. Wealth accumulation $(\Delta k)$ is given by

$$
\Delta k_{t+1}=\left(\frac{1-\alpha \omega_{\zeta}^{2}}{\beta R^{2}-1}\right) \Delta c_{t+1}-\frac{1}{R-1} \Delta y_{t+1}
$$

thus, risk-sensitive consumers adjust their optimal plan by consuming only a fraction of any increase in income. Assets are reduced when income growth is negative and increased income growth is positive. 
Aggregating (2.20) across all consumers gives the change in aggregate consumption:

$$
\Delta C_{t+1}=\frac{R}{R-1} \frac{\beta R^{2}-1}{\beta R^{2}-\alpha \omega_{\zeta}^{2}}\left[\varepsilon_{t+1}+\left(\rho_{1}-1\right) \frac{\varepsilon_{t}}{1-\rho_{1} L}\right] .
$$

Figure 1 illustrates the response of aggregate consumption growth to an aggregate income shock $\varepsilon_{t+1}$; RS raises the sensitivity of consumption growth to unanticipated changes in aggregate income. Furthermore, the smoothness ratio of aggregate consumption to income is seen to be

$$
\mu=\frac{\operatorname{sd}\left[\Delta C_{t}\right]}{\operatorname{sd}\left[\varepsilon_{t}\right]}=\frac{R}{R-1} \frac{\beta R^{2}-1}{\beta R^{2}-\alpha \omega_{\zeta}^{2}} \sqrt{\frac{2}{1+\rho_{1}}},
$$

where "sd" denotes standard deviation. It is straightforward to prove the following proposition, which is the key point from this section.

Proposition 2. $\frac{\partial \mu}{\partial \alpha}>0$.

Proof. Clearly the first term is increasing in $\alpha$. Then

$$
\frac{\partial \rho_{1}}{\partial \alpha}=R \frac{\omega_{\zeta}^{2}(1-R)}{\left(R-\alpha \omega_{\zeta}^{2}\right)^{2}}<0
$$

Thus, $\mu$ is increasing in $\alpha$.

$\mathrm{RS}$ increases the response of aggregate consumption growth to a change in income, just as it does for individual income. Thus, RS by itself worsens the standard RE PIH model's prediction for the joint behavior of aggregate consumption and income growth by exacerbating the excess smoothness puzzle, and therefore needs to be combined with other assumptions to resolve the anomalies. $^{11}$

\section{Permanent Income Models with Rational Inattention}

We now introduce rational inattention (RI) by assuming the consumer faces information processing constraints; as first suggested in Sims (1998), households have only finite Shannon channel capacity that they can apply to observations of the state of the world. As in Sims (2003), we use the concept of entropy from information theory to characterize the rate of information flow; the reduction in entropy is a natural measure of information flow, where entropy is defined as a measure of the uncertainty about a random variable. ${ }^{12}$ With finite channel capacity,

\footnotetext{
${ }^{11}$ In Hansen, Sargent, and Tallarini (1999), habit persistence is incorporated into the RS version of the PIH model. Hansen and Sargent (2007) use habit persistence and/or adjustment costs.

${ }^{12}$ See Shannon (1948) and Cover and Thomas (1991) for details.
} 
the consumer will choose a signal that reduces the uncertainty of the state variable subject to limitations on the extent that entropy can be reduced. Formally, this idea is described by the information processing constraint

$$
\mathcal{H}\left(s_{t+1} \mid \mathcal{I}_{t}\right)-\mathcal{H}\left(s_{t+1} \mid \mathcal{I}_{t+1}\right) \leq \kappa,
$$

where $\kappa$ is the consumer's channel capacity that imposes an upper bound on the amount of information that can be transmitted via the channel, $\mathcal{H}\left(s_{t+1} \mid \mathcal{I}_{t}\right)$ denotes the entropy of the state prior to observing the new signal at $t+1$, and $\mathcal{H}\left(s_{t+1} \mid \mathcal{I}_{t+1}\right)$ denotes the entropy after observing the new signal. ${ }^{13}$

Given the LQG specification, Sims $(2003,2005)$ showed that $\mathcal{D}_{t}$ is distributed $N\left(\widehat{s}_{t}, \sigma_{t}^{2}\right)$, where $\widehat{s}_{t}=E_{t}\left[s_{t}\right]$ and $\sigma_{t}^{2}=\operatorname{var}_{t}\left[s_{t}\right]$ are the conditional mean and variance of the state variable $s_{t}$, respectively. In models with imperfect observations, the optimal decisions are determined by the perceived state, rather than the actual state. Computational difficulties arise in RI models because the perceived state is the distribution of the actual state variable conditional on the information set available at time $t, \mathcal{I}_{t}$, and as we have noted already this object typically has high dimension. Fortunately, given the LQG specification and the Gaussian distribution of $s_{t}$, the first two moments, $\widehat{s}_{t}$ and $\sigma_{t}^{2}$, are enough to characterize the perceived state. In addition, as we will show, the problem will simplify further because $\sigma_{t}^{2}$ converges to a constant in the stochastic steady state.

The constraint (3.1) can be rewritten as

$$
\log \left|\psi_{t}^{2}\right|-\log \left|\sigma_{t+1}^{2}\right| \leq 2 \kappa
$$

where $\sigma_{t+1}^{2}=\operatorname{var}_{t+1}\left[s_{t+1}\right]$ and $\psi_{t}^{2}=\operatorname{var}_{t}\left[s_{t+1}\right]=R^{2} \sigma_{t}^{2}+\operatorname{var}_{t}\left[\zeta_{t+1}\right]$ are the posterior and prior variance of the state variable, $s_{t+1}$, respectively. ${ }^{14}$ As shown in Sims (2003), in any univariate case, this information constraint completes the characterization of the optimization problem with RI and the model can be solved explicitly. Furthermore, with a finite capacity $\kappa$ the optimizing consumer will choose a signal that reduces the conditional variance of $s_{t+1}$ by a limited amount. Hence, (3.2) must be binding for the optimizing consumer:

$$
\log \left|\psi_{t}^{2}\right|-\log \left|\sigma_{t+1}^{2}\right|=2 \kappa
$$

\footnotetext{
${ }^{13}$ We regard $\kappa$ as a technological parameter. If the base for logarithms is 2 , the unit used to measure information flow is a 'bit', and if we use the natural logarithm $e$, the unit is a 'nat'. 1 nat is equal to $\log _{2} e=1.433$ bits. We will use the nat in this paper. Lewis (2006) studies a model where households can increase their channel capacity by reducing their leisure; we have not investigated whether that model can be mapped into the LQ-Gaussian framework.

${ }^{14}$ Note that here we use the fact that the entropy of a Gaussian random variable is equal to half of its logarithm variance plus some constant term.
} 
It is straightforward to show that in the univariate case (3.3) has a steady state $\bar{\sigma}^{2}$. In that steady state, $\sigma_{t}^{2}=\bar{\sigma}^{2}=\frac{\omega_{\zeta}^{2}}{\exp (2 \kappa)-R^{2}}$, where $\omega_{\zeta}^{2}=\operatorname{var}_{t}\left[\zeta_{t+1}\right]=\left(\frac{R}{R-1}\right)^{2} \omega_{\varepsilon}^{2}+\omega_{\epsilon}^{2}$, and the consumer behaves as if he is observing a noisy signal, $s_{t+1}^{*}=s_{t+1}+\xi_{t+1}$, where $\xi_{t+1}$ is the iid endogenous noise and its variance, $\varrho_{t}^{2}=\operatorname{var}_{t}\left[\xi_{t+1}\right]$, is determined by the usual updating formula of the variance of a Gaussian distribution:

$$
\sigma_{t+1}^{2}=\psi_{t}^{2}-\psi_{t}^{2}\left(\psi_{t}^{2}+\varrho_{t}^{2}\right)^{-1} \psi_{t}^{2}
$$

Thus, in the steady state we have

$$
\varrho_{t}^{2}=\bar{\varrho}^{2}=\operatorname{var}_{t}\left[\xi_{t+1}\right]=\frac{\left(\omega_{\zeta}^{2}+R^{2} \bar{\sigma}^{2}\right) \bar{\sigma}^{2}}{\omega_{\zeta}^{2}+\left(R^{2}-1\right) \bar{\sigma}^{2}}
$$

For simplicity, we assume that initially the model economy is at the steady state, implying $s_{0} \mid \mathcal{I}_{0} \sim N\left(\widehat{s}_{0}, \bar{\sigma}^{2}\right)$.

To draw attention to the value of reducing the multivariate PIH model to a univariate one we note that RI with multiple state variables requires a second constraint:

$$
\Psi_{t}-\Sigma_{t} \succeq 0
$$

which is to be read as 'the matrix defined as the difference between the posterior and the prior element-by-element must be positive semidefinite.' We term this constraint a 'no subsidization' condition, as it implies that an agent cannot reduce one variable's entropy by more than $\kappa$ by increasing the entropy of another. This constraint takes the form of restrictions on eigenvalues and thus is not linear except in the univariate case where it is unnecessary. ${ }^{15}$ As a result, the multivariate version of the RI PIH model does not preserve the optimality of Gaussian noise; we therefore exploit the state reduction presented above and use permanent income as the unique state variable.

Luo (2007) contains a complete discussion of the basic linear-quadratic-Gaussian RI model, so we proceed by introducing two models that generalize the RI model to permit precautionary effects. The first, denoted RS-RI, introduces the risk sensitivity motive that we discussed in the previous section. The second, denoted RB-RI, assumes that agents have a demand for robust decision rules (those that are good against a wide range of misspecifications in the model they use for forecasting income movements). We lay out each model and solve it in this section.

\footnotetext{
${ }^{15}$ It is also likely that some variables would generate corner solutions where their entropy is not reduced at all, further exacerbating the nonlinearity of the constraint.
} 


\subsection{RS-RI Model}

The household problem is characterized by the Bellman equation

$$
\widehat{v}\left(\widehat{s}_{t}\right)=\max _{c_{t}}\left\{-\frac{1}{2}\left(c_{t}-\bar{c}\right)^{2}+\beta \mathcal{R}_{t}\left[\widehat{v}\left(\widehat{s}_{t+1}\right)\right]\right\}
$$

subject to the budget constraint, (2.6), and the Kalman filter equation

$$
\widehat{s}_{t+1}=(1-\theta) R\left(\widehat{s}_{t}-c_{t}\right)+\theta\left(s_{t+1}+\xi_{t+1}\right) .
$$

The distorted expectation operator is now given by

$$
\mathcal{R}_{t}\left[\widehat{v}\left(\widehat{s}_{t+1}\right)\right]=-\frac{1}{\alpha} \log E_{t}\left[\exp \left(-\alpha \widehat{v}\left(\widehat{s}_{t+1}\right)\right)\right]
$$

$s_{0} \mid \mathcal{I}_{0} \sim N\left(\widehat{s}_{0}, \bar{\sigma}^{2}\right), \widehat{s}_{t}=E_{t}\left[s_{t}\right]$ is the perceived state variable, $\theta$ is the optimal weight on the new observation of the state, and $\xi_{t+1}$ is the endogenous noise. The optimal choice of the weight $\theta$ is given by

$$
\begin{aligned}
\theta(\kappa) & =\frac{\exp (2 \kappa)-1}{\exp (2 \kappa)} \in[0,1] \\
\theta^{\prime}(\kappa) & =2 \exp (-2 \kappa)>0 ;
\end{aligned}
$$

therefore, we choose to parameterize RI using $\theta$ directly. Furthermore,

$$
\lim _{\kappa \rightarrow \infty} \theta(\kappa)=1
$$

so that we nest the standard RS specification as a special case of our model. To make economic sense in subsequent results we impose the condition $\theta>\frac{R^{2}-1}{R^{2}}>\frac{R-1}{R}$, which implies that agents must be able to process some minimum amount of information. This condition is similar to one imposed in Luo and Young (2007) that guarantees an agent with higher capacity optimally chooses a noise process with lower variance; we regard this requirement to be intuitively reasonable. ${ }^{16}$

The following proposition summarizes the solution to the RI-RS model.

Proposition 1. Given finite channel capacity $\kappa$ and the degree of risk-sensitivity $\alpha$, the value

\footnotetext{
${ }^{16}$ This assumption is not needed in fully nonlinear versions of the RI model, suggesting an important breakdown of the linear-quadratic setup as channel capacity gets very small.
} 
function of a risk-sensitive consumer under $R I$ is

$$
\begin{aligned}
\widehat{v}\left(\widehat{s}_{t}\right) & =-\frac{\beta R^{2}-1}{2 \beta R^{2}-2 \alpha \omega_{\eta}^{2}} \widehat{s}_{t}^{2}+\frac{R\left(1-\beta R^{2}\right)}{\left(\beta R^{2}-\alpha \omega_{\eta}^{2}\right)(R-1)} \widehat{c}_{s_{t}}- \\
& {\left[\frac{1}{2} \frac{R^{2}\left(\beta R^{2}-1\right)}{(R-1)^{2}\left(\beta R^{2}-\alpha \omega_{\eta}^{2}\right)} \bar{c}^{2}-\frac{\beta}{1-\beta} \frac{1}{2 \alpha} \log \left(1-2 \alpha A \omega_{\eta}^{2}\right)\right], }
\end{aligned}
$$

the consumption function is

$$
c_{t}=\frac{\beta R^{2}-1}{\beta R^{2}-\alpha \omega_{\eta}^{2}} \widehat{s}_{t}+\frac{\left(1-\alpha \omega_{\eta}^{2}\right)(R-1)+1-\beta R^{2}}{\left(\beta R^{2}-\alpha \omega_{\eta}^{2}\right)(R-1)} \bar{c}
$$

actual permanent income evolves according to

$$
s_{t+1}=R s_{t}-R \frac{\beta R^{2}-1}{\beta R^{2}-\alpha \omega_{\eta}^{2}} \widehat{s}_{t}-R \frac{\left(1-\alpha \omega_{\eta}^{2}\right)(R-1)+1-\beta R^{2}}{\left(\beta R^{2}-\alpha \omega_{\eta}^{2}\right)(R-1)} \bar{c}+\zeta_{t+1},
$$

and perceived permanent income evolves according to

$\widehat{s}_{t+1}=\frac{(1-\theta) R\left(1-\alpha \omega_{\eta}^{2}\right)-\theta R\left(\beta R^{2}-1\right)}{\beta R^{2}-\alpha \omega_{\eta}^{2}} \widehat{s}_{t}+\theta R s_{t}-R \frac{\left(1-\alpha \omega_{\eta}^{2}\right)(R-1)+1-\beta R^{2}}{\left(\beta R^{2}-\alpha \omega_{\eta}^{2}\right)(R-1)} \bar{c}+\theta\left(\zeta_{t+1}+\xi_{t+1}\right)$,

where

$$
\omega_{\eta}^{2}=\operatorname{var}\left[\eta_{t+1}\right]=\frac{\theta}{1-(1-\theta) R^{2}} \omega_{\zeta}^{2}
$$

and

$$
\eta_{t+1}=\theta\left[\frac{\zeta_{t+1}}{1-(1-\theta) R L}+\left(\xi_{t+1}-\frac{\theta R \xi_{t}}{1-(1-\theta) R L}\right)\right]
$$

Proof. See Appendix 7.3.

To interpret these expressions, we remind the reader that $\xi_{t}$ is the noise shock induced by $\mathrm{RI}$ and $\zeta_{t}$ is the innovation to permanent income. $\eta_{t+1}$ will turn out to be the stochastic component in the growth rate of consumption (see below); unlike the basic RS model, $\eta_{t+1}$ contains a forecastable stochastic component due to the presence of the $\mathrm{MA}(\infty)$ term. There are two special cases of interest that are nested in our specification: if $\alpha=0$ we obtain the same expressions as in Luo (2007) (the basic RI model), and if $\theta=1$ we obtain $\eta_{t+1}=\zeta_{t+1}$ (the basic RS model).

Note that the permanent income shock $\zeta_{t}$ and the noise shock $\xi_{t}$ have the same effect on $\widehat{s}_{t+1}$ (they both increase perceived permanent income by $\theta \leq 1$ ) but only $\zeta_{t}$ affects $s_{t+1}$ (it increases by 1 ); because the variance of the noise shock will be decreasing in $\theta$, agents with high processing capacity will end up with $\widehat{s}_{t}$ tracking $s_{t}$ quite closely. The consumption function shows that $\theta$ affects both the MPC out of the perceived state variable $\frac{\beta R^{2}-1}{\beta R^{2}-\alpha \omega_{\eta}^{2}}$ and the precautionary savings 
increment $\frac{\left(1-\alpha \omega_{\eta}^{2}\right)(R-1)+1-\beta R^{2}}{\left(\beta R^{2}-\alpha \omega_{\eta}^{2}\right)(R-1)}$ only through the variance of the noise distribution. With finite capacity $\omega_{\eta}^{2}>\omega_{\zeta}^{2}$ and it then follows that $\omega_{\eta}^{2}$ is decreasing in $\theta$.

Proposition 2. $\frac{\partial \omega_{\eta}^{2}}{\partial \theta}<0$.

Proof. By simple calculation we obtain

$$
\frac{\partial \omega_{\eta}^{2}}{\partial \theta}=\frac{\left(1-R^{2}\right) \omega_{\zeta}^{2}}{\left(1-(1-\theta) R^{2}\right)^{2}}<0
$$

because $R>1$ and $1-(1-\theta) R^{2}>0$.

Thus, both the MPC out of the perceived state variable (the responsiveness of $c_{t}$ to $\widehat{s}_{t}$ ) and the precautionary savings increment (the intercept of the consumption profile) are decreasing in $\theta$ for fixed $\alpha$, meaning that agents with lower capacity save a larger fraction of any perceived income increase. Figure 2 illustrates how the combination of $(\alpha, \theta)$ affects the MPC out of financial and human wealth (which are equal here) when $R=1.01$. The relative risk aversion expression is now

$$
r\left(\widehat{s}_{t}\right)=\frac{1}{\frac{R}{R-1} \frac{\bar{c}}{\hat{s}_{t}}-1} ;
$$

the key difference is that risk aversion depends not on how far actual permanent income is from the bliss point but rather how far perceived permanent income is from the bliss point. An RS-RI agent is more risk averse than a standard RS agent whenever $\widehat{s}_{t}>s_{t}$, which occurs when the agent receives negative shocks to $\zeta$ or positive shocks to $\xi$.

Since agents with low capacity are very concerned about the confluence of low permanent income and high consumption (meaning they believe their permanent income is high so they consume a lot and then their new signal indicates that in fact their permanent income was low), they take actions which reduce the probability of this bad event - they save more. ${ }^{17}$ The strength of the precautionary effect is positively related to the amount of uncertainty regarding the true level of permanent income, and this uncertainty increases as $\theta$ gets smaller. Figure 3 illustrates the effect of $(\alpha, \theta)$ on the precautionary savings increment. In the absence of risk sensitivity ( $\alpha=0$ ) the precautionary savings increment is zero independent of $\theta$; if $\alpha>0$, however, precautionary savings is decreasing in $\theta$, so that agents with lower capacity will consume less on average. $^{18}$

\footnotetext{
${ }^{17} \mathrm{~A}$ more complete discussion of precautionary savings in an RI model can be found in Batchuluun, Luo, and Young (2007).

${ }^{18}$ Precautionary savings in this model is different from the precautionary savings that arises when there is an interaction between exogenous income risk and convex marginal utility. Precautionary savings arises here because RI agents cannot use all available information to make decision, so it does not require convexity of the marginal utility function.
} 


\subsubsection{Individual Dynamics}

The change in individual consumption in the RI-RS economy can be written as

$\Delta c_{t+1}=\frac{R-\beta R^{2}+(1-R) \alpha \omega_{\eta}^{2}}{\beta R^{2}-\alpha \omega_{\eta}^{2}}\left(c_{t}-\bar{c}\right)+\theta R \frac{\beta R^{2}-1}{\beta R^{2}-\alpha \omega_{\eta}^{2}}\left(\frac{(1-\theta) \zeta_{t}-\theta \xi_{t}}{1-(1-\theta) R L}\right)+\theta \frac{\beta R^{2}-1}{\beta R^{2}-\alpha \omega_{\eta}^{2}}\left(\zeta_{t+1}+\xi_{t+1}\right)$

so that

$$
\Delta c_{t}=\theta \frac{\beta R^{2}-1}{\beta R^{2}-\alpha \omega_{\eta}^{2}}\left\{\sum_{j=0}^{\infty} \Gamma_{j} \zeta_{t-j}+\sum_{j=0}^{\infty} \Gamma_{j} \xi_{t-j}-R \sum_{j=0}^{\infty} \Gamma_{j} \xi_{t-1-j}\right\}+\frac{R-\beta R^{2}+(1-R) \alpha \omega_{\eta}^{2}}{\beta R^{2}-\alpha \omega_{\eta}^{2}} \bar{c}
$$

where $\rho_{1}=\frac{R-\alpha \omega_{\eta}^{2} R}{\beta R^{2}-\alpha \omega_{\eta}^{2}} \in(0,1), \rho_{2}=(1-\theta) R \in(0,1)$, and

$$
\Gamma_{j}=\sum_{k=0}^{j}\left(\rho_{1}^{j-k} \rho_{2}^{k}\right)-\sum_{k=0}^{j-1}\left(\rho_{1}^{j-1-k} \rho_{2}^{k}\right)
$$

Thus, consumption growth is seen to be a weighted average of all past permanent income and noise shocks.

Wealth accumulation under RI is

$$
\Delta k_{t+1}=\frac{1-\alpha \omega_{\eta}^{2}}{\beta R^{2}-1} \Delta c_{t+1}-\frac{1}{R-1} \Delta y_{t+1}+\Delta\left(s_{t+1}-\widehat{s}_{t+1}\right)
$$

under RI the actual savings of risk-sensitive consumers responds to unperceived changes in the true state relative to the perceived one.

Figure 4 plots the responses of consumption to the aggregate permanent income shock $\varepsilon_{t}$. It clearly shows that RI has a similar qualitative effect on consumption in the standard RE model and the RS model ${ }^{19}$ quantitatively, RI has larger impacts on consumption in the RS model because the deviation of the asymptote from that for the standard RE case is larger in this case. With a stronger preference for risk-sensitivity, the precautionary savings increment is larger and thus an income shock that is initially undetected would have larger impacts on consumption during the adjustment process. In addition, we note that the immediate response of consumption to a shock to permanent income is given by

$$
\operatorname{SR}\left(\varepsilon_{t}\right)=\theta \frac{\beta R^{2}-1}{\beta R^{2}-\alpha \omega_{\eta}^{2}}
$$

This expression is increasing in $\alpha$, implying that the more risk-sensitive the household the

\footnotetext{
${ }^{19}$ That is, consumption reacts gradually to income shocks, with monotone adjustments to the corresponding asymptote.
} 
more consumption responds initially to changes in permanent income. It is also increasing in $\theta$, implying that agents with lower capacity have smaller initial responses, so that RI and RS have opposing effects. In Figure 5 we plot the short-run responses of consumption growth as a function of $\theta$ for various values of $\alpha$.

With RI agents respond slowly, similar to their response if they faced convex adjustment costs for their wealth; one resulting implication is that the short-run and long-run responses of consumption are different. The long-run response is given by

$$
\operatorname{LR}\left(\varepsilon_{t}\right)=\frac{\partial\left(\sum_{s=0}^{T} \Delta c_{t+s}\right)}{\partial \varepsilon_{t}}=\theta \frac{\beta R^{2}-1}{\beta R^{2}-\alpha \omega_{\eta}^{2}} \sum_{k=0}^{T}\left(\rho_{1}^{T-k} \rho_{2}^{k}\right)
$$

Note that when $\alpha=0$ and $\rho_{1}=1$ and taking the limit as $T \rightarrow \infty$ this expression reduces to the result reported in Luo (2007):

$$
\operatorname{LR}\left(\varepsilon_{t}\right)=\frac{\theta}{1-\rho_{2}} .
$$

It is clear from the above two expressions that RS has two opposite impacts on the long-run responses of consumption to income shocks under RI. First, $\alpha$ increases the long-run response by a factor $\theta \frac{R}{R-\alpha \omega_{\eta}^{2}}$; second, it reduces the long-run response by a factor

$$
\lim _{T \rightarrow \infty} \sum_{k=0}^{T}\left(\rho_{1}^{T-k} \rho_{2}^{k}\right)=0<\frac{1}{1-\rho_{2}}
$$

because $\left|\rho_{1}\right|<1$.

Furthermore, the volatility of individual consumption growth is

$\operatorname{var}\left[\Delta c_{t}\right]=\left(\theta \frac{R-1}{R-\alpha \omega_{\eta}^{2}}\right)^{2}\left\{\sum_{j=0}^{\infty} \Gamma_{j}^{2} \omega_{\zeta}^{2}+\sum_{j=0}^{\infty}\left[\Gamma_{j}-(1+R) \sum_{k=0}^{j-1}\left(\rho_{1}^{j-1-k} \rho_{2}^{k}\right)+\sum_{k=0}^{j-2}\left(\rho_{1}^{j-1-k} \rho_{2}^{k}\right)\right]^{2} \omega_{\xi}^{2}\right\}$.

Intuitively, this expression should be decreasing in $\theta$ and increasing in $\alpha$. With lower $\theta$, agents have less channel capacity and therefore choose more volatile noise shocks, leading to changes in consumption that are more sensitive to changes in perceived permanent income.

\subsubsection{Aggregate Dynamics}

Since the expression for the change in individual consumption (3.12) permits exact aggregation, we can obtain the change in aggregate consumption as

$$
\Delta C_{t}=\theta \frac{\beta R^{2}-1}{\beta R^{2}-\alpha \omega_{\eta}^{2}}\left\{\sum_{j=0}^{\infty} \Gamma_{j} \varepsilon_{t-j}+\sum_{j=0}^{\infty} \Gamma_{j} E^{i}\left[\xi_{t-j}\right]-R \sum_{j=0}^{\infty} \Gamma_{j} E^{i}\left[\xi_{t-1-j}\right]\right\}
$$


where $i$ denotes a particular individual and $E^{i}[\cdot]$ is the population average. This expression shows that even if every consumer only faces the common shock $\varepsilon$, the RI economy still has heterogeneity since each consumer faces the idiosyncratic noise induced by finite channel capacity. As argued in Sims (2003), although the randomness in an individual's response to aggregate shocks will be idiosyncratic because it arises from the individual's information-processing constraint, there is likely a significant common component; provided that agents face similar needs for coding macroeconomic information, they will rely on common sources and generate aggregate noise shocks. Existing theory does not provide a way to determine the common component of the noise term; we can only state that the common term $E^{i}\left[\xi_{t}^{i}\right]$ is between 0 and the part of the idiosyncratic error $\xi_{t}$ generated by the aggregate income shock $\varepsilon_{t}$.

In order to simplify expressions we consider the case where all noises are idiosyncratic (so that individuals live on isolated islands and do not interact with each other directly or indirectly via conversation, imitation, newspapers, or other media); in this special case the change in aggregate consumption can be written as

$$
\Delta C_{t}=\theta \frac{\beta R^{2}-1}{\beta R^{2}-\alpha \omega_{\eta}^{2}}\left\{\sum_{j=0}^{\infty} \Gamma_{j} \varepsilon_{t-j}\right\} .
$$

Therefore, the volatility of aggregate consumption relative to income can be written as

$$
\mu=\frac{\operatorname{sd}\left[\Delta C_{t}\right]}{\operatorname{sd}\left[\varepsilon_{t}\right]}=\left(\theta \frac{\beta R^{2}-1}{\beta R^{2}-\alpha \omega_{\eta}^{2}}\right) \sqrt{\sum_{j=0}^{\infty} \Gamma_{j}^{2}},
$$

which means that RI reduces the volatility of aggregate consumption growth in the risk-sensitive PIH model. Figure 5 illustrates how the combination of RI and RS affects the smoothness ratio of aggregate consumption. It is clear that incorporating RI into the risk-sensitive PIH model can reduce the volatility of aggregate consumption and thus make the model better explain the data in this aspect. For example, when $\alpha=5 \times 10^{-6}, \omega_{\varepsilon}=5.6, R=1.01$, and $\theta=0.15$, the excess smoothness ratio is about 0.47 , very close to its empirical counterpart in the US data.

\subsection{Robust-RI Model}

We now consider the connections between risk sensitivity and robustness. Hansen and Sargent (2007) demonstrate a deep connection between the choices made by an agent that is risk-sensitive and one that has a preference for robustness. An agent with a preference for robust decision rules considers a range of models and makes decisions that maximize utility given the worst possible model. Following Hansen and Sargent (2007), the robust PIH problem with inattentive 
consumers can be written as

$$
\widehat{v}\left(\widehat{s}_{t}\right)=\max _{c_{t}} \min _{\nu_{t}}\left\{-\frac{1}{2}\left(c_{t}-\bar{c}\right)^{2}+\beta E_{t}\left[\vartheta \nu_{t}^{2}+\widehat{v}\left(\widehat{s}_{t+1}\right)\right]\right\}
$$

subject to the flow budget constraint

$$
s_{t+1}=R\left(s_{t}-c_{t}\right)+\zeta_{t+1}+\omega_{\zeta} \nu_{t}
$$

and the Kalman filter equation

$$
\widehat{s}_{t+1}=(1-\theta)\left[R\left(\widehat{s}_{t}-c_{t}\right)+\omega_{\zeta} \nu_{t}\right]+\theta\left(s_{t+1}+\xi_{t+1}\right)
$$

where $\nu_{t}$ distorts the mean of the innovation and $\vartheta>0$ controls how bad the error can be. ${ }^{20}$ After substituting (3.21) into (3.22), the Kalman filter equation can be rewritten as

$$
\widehat{s}_{t+1}=R\left(\widehat{s}_{t}-c_{t}\right)+\omega_{\zeta} \nu_{t}+\eta_{t+1} .
$$

where $\eta_{t+1}=\theta R\left(s_{t}-\widehat{s}_{t}\right)+\theta\left(\zeta_{t+1}+\xi_{t+1}\right)$.

The following proposition summarizes the solution to the RB-RI model.

Proposition 3. Given $\vartheta$ the value function of an agent with preference for robustness under $R I$ is given by

$$
\begin{aligned}
\widehat{v}\left(\widehat{s}_{t}\right) & =-\frac{\beta R^{2}-1}{2 \beta R^{2}-\omega_{\zeta}^{2} / \vartheta} \widehat{s}_{t}^{2}-\frac{R}{R-1} \frac{\beta R^{2}-1}{\beta R^{2}-\omega_{\zeta}^{2} /(2 \vartheta)} \widehat{c}_{t}- \\
& \left(\frac{R^{2}\left(\beta R^{2}-1\right)}{2\left(\beta R^{2}-\omega_{\zeta}^{2} /(2 \vartheta)\right)(R-1)^{2}} \bar{c}^{2}+\frac{\beta}{1-\beta} \frac{\beta R^{2}-1}{2 \beta R^{2}-\omega_{\zeta}^{2} / \vartheta} \omega_{\eta}^{2}\right),
\end{aligned}
$$

the consumption function is

$$
c_{t}=\frac{\beta R^{2}-1}{\beta R^{2}-\omega_{\zeta}^{2} /(2 \vartheta)} \widehat{s}_{t}+\frac{\left(1-\omega_{\zeta}^{2} /(2 \vartheta)\right)(R-1)+1-\beta R^{2}}{\left(\beta R^{2}-\omega_{\zeta}^{2} /(2 \vartheta)\right)(R-1)} \bar{c}
$$

\footnotetext{
${ }^{20}$ Formally, this setup is a game between the decision-maker and a malevolent nature that chooses the distortion process $\nu_{t} . \quad \vartheta \geq 0$ is a penalty parameter that restricts attention to a limited class of distortion processes; it can be mapped into an entropy condition that implies agents choose rules that are robust against processes which are close to the trusted one.
} 
the optimal worst-case distribution is given by

$$
\nu_{t}=\frac{\omega_{\zeta}^{2}}{2 \vartheta} \frac{\beta R^{2}-1}{\beta R\left(\beta R^{2}-\omega_{\zeta}^{2} /(2 \vartheta)\right)} \widehat{s}_{t}+\frac{\omega_{\zeta}^{2}}{2 \vartheta}\left(\frac{R}{R-1}\right) \frac{\left(\beta R^{2}-1\right)}{\beta R\left(\beta R^{2}-\omega_{\zeta}^{2} /(2 \vartheta)\right)} \bar{c} .
$$

Proof. See Appendix 7.4.

Equation (3.25) displays an important property - it is independent of $\theta$. Thus, in the RB-RI model limited channel capacity does not affect consumption except through changes in the level of perceived permanent income. The same property holds for (3.26); the worst-case distribution does not directly depend on the channel capacity. The value function therefore only directly depends on $\theta$ through the constant term. Individual and aggregate dynamics of consumption under robustness are very similar to those under RS, so we omit examining the dynamics under robustness.

\section{Observational Equivalence}

In this section we detail the observational equivalence results that connect the RI, RS-RI, and RB-RI models. All three models are capable of producing the same consumption-savings decisions for some combination of parameters $(\alpha, \beta, \vartheta, \theta)$, rendering them fundamentally unidentified by this data alone. Our purpose is to provide intuition about why the observational equivalence result obtains in three settings - between RI and RS-RI, between RI and RB-RI, and between RS-RI and RB-RI.

\subsection{Observational Equivalence Between RI and RS-RI}

Hansen, Sargent, and Tallarini (1999) show that as far as the quantity observations on consumption and investment are concerned, the risk-sensitive version $(\alpha>0, \widetilde{\beta})$ of the PIH model is observationally equivalent to the standard version $(\alpha=0, \beta)$ of the PIH model for a unique pair of discount factors. ${ }^{21}$ However, the two models have different implications for asset prices. In this section we show that holding all parameters constant except the pair $(\alpha, \beta)$, the RI version of the PIH model with risk-sensitive consumers $(\alpha>0$ and $\beta R<1)$ is observationally equivalent to the standard RI version of the model $(\alpha=0$ and $\beta R=1)$, extending the observational equivalence result to a broader class of models. To do so, we fix $R$ and assume that $\beta$ differs across the two settings; thus, $\beta^{R S, R I}$ is the discount factor needed in the RS-RI model to achieve OE with the RI model where $\beta R=1$.

\footnotetext{
${ }^{21}$ Hansen, Sargent, and Tallarini (1999) derive the observational equivalence result by fixing all parameters, including $R$, except for the pair $(\alpha, \beta)$.
} 
Proposition 1. Let

$$
\beta^{R S, R I}=\frac{R-(R-1) \alpha \omega_{\eta}^{2}}{R^{2}}<\frac{1}{R} .
$$

Then consumption and savings are identical in the RI and RS-RI models.

Proof. See Appendix 7.5.

This proposition is straightforward to prove by simply setting the coefficients in the consumption function equal and solving for the discount factor. We show in the appendix that the same outcome is obtained by setting the slope and curvature coefficients in the value functions equal. (4.1) implies that the required discount factor is decreasing as a function of $\alpha$ and increasing as a function of $\theta$ :

$$
\begin{aligned}
\frac{\partial}{\partial \alpha}\left(\beta^{R S, R I}\right) & =-\frac{(R-1) \omega_{\eta}^{2}}{R^{2}}<0 \\
\frac{\partial}{\partial \theta}\left(\beta^{R S, R I}\right) & =-\frac{(R-1) \alpha}{R^{2}} \frac{\partial \omega_{\eta}^{2}}{\partial \theta}>0 .
\end{aligned}
$$

Holding fixed $\theta$, an agent who is more sensitive to risk (higher $\alpha$ ) will save more; thus, to match an agent who is not sensitive to risk that agent must be less patient. Similarly, holding fixed $\alpha$ an agent with a higher channel capacity (higher $\theta$ ) will save less, requiring them to be more patient to match the saving of an agent who is risk sensitive but has lower channel capacity. Note that $\frac{\partial}{\partial \theta}\left(\beta^{R S, R I}\right)=0$ if $\alpha=0$, so that the discount factor would not need to be adjusted if agents are not risk sensitive; this result simply restates the result in Luo (2007) and arises because RI does not alter consumption-savings plans when $\alpha=0$. Figure 6 plots the locus of OE between $(\alpha, \beta)$ for various different values of $\theta$ (we fix $R=1.01$ ).

\subsection{Observational Equivalence Between RI and RB-RI}

We now turn to a comparison of RI and RB-RI. As above, we can derive an expression that links the discount factor in the RB-RI model to the robustness parameter $\vartheta$ in such a way that the agent would make the same choices in the RI and RB-RI models.

Proposition 2. Let

$$
\beta^{R B, R I}=\frac{R-(R-1) \omega_{\zeta}^{2} /(2 \vartheta)}{R^{2}}<\frac{1}{R} .
$$

Then consumption and savings are identical in the $R I$ and $R B-R I$ models.

Proof. See Appendix 7.5.

Here, the discount factor needed in the RB-RI model does not depend on the channel capacity parameter $\theta$; this independence is a consequence of the previously-noted independence of 
consumption in this model. It is immediate that the discount factor is an increasing function of $\vartheta$ :

$$
\frac{\partial}{\partial \vartheta}\left(\beta^{R B, R I}\right)=\frac{R-1}{R^{2}} \frac{\omega_{\zeta}^{2}}{2 \vartheta^{2}}>0
$$

An agent with higher $\vartheta$ is less concerned about model misspecification and therefore tends to save less (equivalently, they consider only models that are very close to the trusted one, leading to less distortion and therefore less additional saving); in order to match the behavior of an agent who does not require robust decisions the robust agent must be made more patient. If $\vartheta=\infty$ then $\frac{\partial}{\partial \vartheta}\left(\beta^{R B, R I}\right)=0$; again, this result matches that found in Luo (2007).

\subsection{Observational Equivalence Between RS-RI and RB-RI}

We now turn the final comparisons, where provide settings for $(\alpha, \beta, \theta, \vartheta)$ that jointly produce observational equivalence. These results imply that there exists a fundamental lack of identification between the class of models we are considering here (linear-quadratic-Gaussian models); it would be impossible to distinguish between any of them using only consumption-savings decisions. $^{22}$

Proposition 3. Let the following expression hold:

$$
\frac{\alpha \theta}{1-(1-\theta) R^{2}}=\frac{1}{2 \vartheta}
$$

Then consumption and savings are identical in the RS-RI and RB-RI models.

Proof. See Appendix (7.5).

To interpret these expressions we fix $\beta$ at some common value, since it does not play a role here. An agent who is risk-sensitive $(\alpha>0)$ and suffers from finite Shannon channel capacity $(\theta<1)$ will make the same choices as an agent who is concerned with robustness $(\vartheta<\infty)$. The "required" $\vartheta$ is given by

$$
\vartheta=\frac{1-(1-\theta) R^{2}}{2 \alpha \theta}
$$

where

$$
\begin{aligned}
& \frac{\partial \vartheta}{\partial \theta}=\frac{R^{2}-1}{2 \alpha \theta^{2}}>0 \\
& \frac{\partial \vartheta}{\partial \alpha}=-\frac{1-(1-\theta) R^{2}}{2 \alpha^{2} \theta}<0
\end{aligned}
$$

\footnotetext{
${ }^{22}$ By extension, if we interpret our model as a model of capital accumulation with a linear technology, it is impossible to distinguish the models using only consumption and investment data.
} 
That is, if we compare two agents with the same $\alpha$, the one with higher channel capacity will behave the same as an agent who is less concerned with model misspecification. If we compare two agents with the same $\theta$, the one with higher risk sensitivity will behave the same as an agent who is more concerned with model misspecification.

Another observational equivalence result arises if we fix $(\alpha, \vartheta)$ for two agents who have the same $\theta$. Then, there exists a pair of discount factors, $\beta^{R I, R S}$ and $\beta^{R I, R B}$, such that the two agents choose the same consumption plans.

Proposition 4. Let the two discount factors satisfy the relation

$$
\beta^{R I, R B}=\frac{\left(1-R^{2} \beta^{R I, R S}\right) \omega_{\zeta}^{2}+2 \vartheta\left(R^{2} \beta^{R I, R S}-\alpha \omega_{\eta}^{2}\right)}{2 \vartheta R^{2}\left(1-\alpha \omega_{\eta}^{2}\right)} .
$$

Then consumption and savings are identical in the $R S-R I$ and $R B-R I$ models.

\section{Welfare Costs From RI}

We present here the welfare cost of RI - what an agent would pay to increase $\kappa$ to $\infty$ (so that the optimal choice is $\theta=1$ ). This calculation is defined as the difference between the value function with and without information-processing constraints, expressed in terms of a money metric. Luo (2007) shows that the welfare costs of RI are fairly small in the certainty equivalent environment; our interest here is assessing how these costs differ in the RS-RI and RB-RI models. ${ }^{23}$ To make the comparisons meaningful, we restrict our attention to combinations of preferences that imply observational equivalence - that is, do agents who look the same (but for different reasons) suffer the same welfare losses from information-processing capacity limitations?

\subsection{Welfare Costs in RS-RI and RB-RI}

We assume for this section that $(\alpha, \vartheta)$ are such that OE obtains between the two models (note that $\mathrm{OE}$ requires that $\alpha$ depend on $\theta$ ). Within this class we can derive the lifetime utility in the RS-RI model,

$$
\widehat{v}^{R S}\left(\widehat{s}_{t}\right)=-\frac{R-1}{2 R} \widehat{s}_{t}^{2}+\widehat{c}_{t}-\frac{1}{2}\left(\frac{R}{R-1} \bar{c}^{2}-\frac{\beta}{1-\beta} \frac{1}{\alpha} \log \left(1-\frac{R-1}{R} \alpha \omega_{\eta}^{2}\right)\right),
$$

and in the RB-RI model,

$$
\widehat{v}^{R B}\left(\widehat{s}_{t}\right)=-\frac{R-1}{2 R} \widehat{s}_{t}^{2}+\widehat{c}_{t}-\frac{1}{2}\left(\frac{R}{R-1} \bar{c}^{2}+\frac{\beta}{1-\beta} \frac{R-1}{R} \omega_{\eta}^{2}\right)
$$

\footnotetext{
${ }^{23}$ Our calculation is related to Tallarini (2000), who shows how risk-sensitivity increases the welfare cost of business cycles and the equity premium.
} 
where $\beta=\beta^{R S, R I}=\beta^{R B, R I}$. The only difference between the two functions is the constant term; this equivalence arises because the slope and curvature parameters are pinned down by the equalization of the consumption decisions. We note that

$$
\lim _{\alpha \rightarrow 0}\left\{-\frac{1}{\alpha} \log \left(1-\frac{R-1}{R} \alpha \omega_{\eta}^{2}\right)\right\}=\frac{R-1}{R} \omega_{\eta}^{2}
$$

so that the two expressions are equal if $\alpha=0$. We also note that

$$
\frac{\partial}{\partial \alpha}\left(-\frac{1}{\alpha} \log \left(1-\frac{R-1}{R} \alpha \omega_{\eta}^{2}\right)\right)>0
$$

Thus, we have

$$
-\frac{1}{\alpha} \log \left(1-2 \frac{R-1}{R} \alpha \omega_{\eta}^{2}\right)>\frac{R-1}{R} \omega_{\eta}^{2}
$$

for $\alpha>0$. This result implies that RS households have lower utility than RB agents do, conditional on being observationally equivalent. We summarize the previous discussion as a formal proposition.

Proposition 1. Given the same level of the perceived state $\widehat{s}_{t}$ and assuming (4.3) is satisfied by $(\alpha, \vartheta)$ for fixed $(\theta, \beta)$, it follows that $\widehat{v}^{R S}\left(\widehat{s}_{t}\right)<\widehat{v}^{R B}\left(\widehat{s}_{t}\right)$.

A similar proposition holds in the absence of RI simply by replacing $\omega_{\eta}^{2}$ with $\omega_{\zeta}^{2}$, so that it holds when $\theta=1$. This result implies that the costs of uncertainty are larger for RS agents than RB agents whenever the parameters are such that their observable behavior is identical. Both types of agents respond by saving more than a standard RI agent would; under the OE restriction for parameters, they therefore generate the same consumption path. But RS agents are more averse to the remaining fluctuations in consumption than RB agents are, resulting in higher costs of uncertainty and lower lifetime utility.

Proposition 2. Define the difference between the welfare losses of $R S$ and $R B$ consumers as

$$
\Delta=-\frac{1}{\alpha} \log \left(1-\frac{R-1}{R} \alpha \omega_{\eta}^{2}\right)-\frac{R-1}{R} \omega_{\eta}^{2}
$$

$\Delta$ is decreasing in $\theta$.

Proof. By straightforward differentiation we obtain

$$
\frac{d \Delta}{d \theta}=\frac{d \Delta}{d \omega_{\eta}^{2}} \frac{d \omega_{\eta}^{2}}{d \theta}<0
$$

because

$$
\frac{d \Delta}{d \omega_{\eta}^{2}}=\frac{R-1}{R-(R-1) \alpha \omega_{\eta}^{2}}-\frac{R-1}{R}>0 \text { for } \alpha>0 \text { and } \frac{d \omega_{\eta}^{2}}{d \theta}<0
$$


$\mathrm{RI}$ increases the difference between the welfare losses of $\mathrm{RS}$ and $\mathrm{RB}$ consumers who have the same consumption-savings decisions; as $\theta$ decreases, the cost of RI for the RS agents increases faster than the cost for the RB agents.

\subsection{Quantitative Results}

To compute the welfare losses of RS and RB consumers from RI due to deviating from the first-best RE path, we need the costs of deviating from the RE benchmark. Specifically, we first assume that at the beginning both RS and RB consumers have unlimited capacity and choose the same consumption-savings decisions, that is, the OE under RE holds $(\alpha=1 /(2 \vartheta))$; we then increase the degree of inattention and compute the welfare costs of RI due to deviating from the RE path. After deriving the value functions in all cases, it is straightforward to show that under the OE, $\alpha=1 /(2 \vartheta)$, the expressions for the expected welfare losses from RI are

$$
\begin{aligned}
E[\Delta v(\alpha)] & =\frac{\beta R^{2}-1}{2}\left(\frac{1}{\beta R^{2}-\alpha \omega_{\eta}^{2}} E\left[\widehat{s}_{t}^{2}\right]-\frac{1}{\beta R^{2}-\alpha \omega_{\zeta}^{2}} E\left[s_{t}^{2}\right]\right)+ \\
& \frac{\left(\beta R^{2}-1\right) R \bar{c}}{R-1}\left(\frac{1}{R^{2} \beta-\alpha \omega_{\zeta}^{2}} E\left[s_{t}\right]-\frac{1}{R^{2} \beta-\alpha \omega_{\eta}^{2}} E\left[\widehat{s}_{t}\right]\right)+ \\
& \frac{R^{2}\left(R^{2} \beta-1\right) \bar{c}^{2}}{2(R-1)^{2}}\left(\frac{1}{R^{2} \beta-\alpha \omega_{\eta}^{2}}-\frac{1}{R^{2} \beta-\alpha \omega_{\zeta}^{2}}\right)+\frac{\beta}{1-\beta} \frac{1}{2 \alpha} \log \left(\frac{1-\alpha \omega_{\zeta}^{2} \frac{R^{2} \beta-1}{\beta R^{2}-\alpha \omega_{\zeta}^{2}}}{1-\alpha \omega_{\eta}^{2} \frac{R^{2} \beta-1}{\beta R^{2}-\alpha \omega_{\eta}^{2}}}\right),
\end{aligned}
$$

where $\beta=\frac{R-(R-1) \alpha \omega_{\zeta}^{2}}{R^{2}}$ for the RS-RI model and

$$
E[\Delta v(\vartheta)]=\frac{R-1}{2 R}\left(\operatorname{var}\left(\widehat{s}_{t}\right)-\operatorname{var}\left(s_{t}\right)\right)+\frac{\beta}{1-\beta} \frac{R-1}{2 R}\left(\omega_{\eta}^{2}-\omega_{\zeta}^{2}\right)
$$

where $\beta=\frac{R-(R-1) \omega_{\zeta}^{2} /(2 \vartheta)}{R^{2}}$ for the RB-RI model.

To do quantitative welfare analysis in the models we need to know the level of $E\left[s_{t}\right]$ (note that it is equal to $E\left[\widehat{s}_{t}\right]$ ). First, denote by $\gamma$ the local coefficient of relative risk aversion, which equals

$$
\gamma=\frac{E[y]}{\bar{c}-E[y]}
$$

for the utility function $u(\cdot)$ evaluated at mean income $E[y]$. To calculate the welfare losses due to RI, we set the parameters according to those estimated from post-World War U.S. time series by Hansen, Sargent, and Tallarini (1999) for a PIH model with two-factor endowment process. Specifically, we follow the procedure used in Hansen and Sargent (2004) and use the estimated 
one-factor endowment process as follows

$$
y_{t+1}=0.9992 y_{t}+\varepsilon_{t+1}
$$

and $\varepsilon_{t+1}$ follows an iid process distributed as $N(0,5.6)$. Since the estimated persistence coefficient is difficult to distinguish from 1 , for simplicity we assume that income follows a random walk. Following Hansen, Sargent, and Tallarini (1999) we also set the mean income level $E[y]=16$ and then find the value of the bliss point $\bar{c}$ that generates reasonable relative risk aversion $\gamma$. For example, if $\gamma$ is equal to $1, \bar{c}=2 E[y]=32$. Furthermore, assume that the ratio of mean financial wealth to mean labor income is 5 , that is, $E[w] / E[y]=5 .^{24}$ Since $s_{t}=w_{t}+\frac{1}{R-1} y_{t}$, we have

$$
E\left[s_{t}\right]=\left(5+\frac{1}{R-1}\right) E\left[y_{t}\right] .
$$

We will use this specification when we make quantitative statements.

Following Cochrane (1989), Pischke (1995), and others, we use a money metric to measure the welfare cost of deviating from the standard RS and RB solutions. Specifically, dividing the expected welfare losses $E[\Delta v]=E\left[v\left(s_{t} ; \theta=1\right)-\widehat{v}\left(\widehat{s}_{t} ; \theta<1\right)\right]$ by the marginal utility of a dollar at time $t$ and converting it to dollars per quarter yields

$$
\$ \text { Loss/quarter: } \Delta^{R S}=\frac{R-1}{R} \frac{E[\Delta v(\alpha)]}{u^{\prime}(\bar{y})} \text {, }
$$

and

$$
\$ \text { Loss/quarter: } \Delta^{R B}=\frac{R-1}{R} \frac{E[\Delta v(\vartheta)]}{u^{\prime}(\bar{y})} .
$$

where $\Delta v(\alpha)$ and $\Delta v(\vartheta)$ are defined in (5.5) and (5.6), respectively.

Our purpose in presenting these numerical results is to demonstrate that the welfare losses from RI can in fact be considerably larger for RS agents. To this end, Table 1 reports the ratio of the welfare losses of RS to RB, $\Delta^{R S} / \Delta^{R B}$. We set $\theta=1$ and $\alpha=\frac{1}{2 \vartheta}$ to reflect the OE between robustness and risk-sensitivity in the $\mathrm{RE}$ context and then consider lower values of $\theta$, adjusting parameters appropriately to maintain OE. The costs are uniformly higher for the RS model, and can in fact be much larger (the rightmost column has ratios that approach 8). Similarly, Table 2 reports the ratio of the RI-induced welfare losses in the RS-RI and basic RI models for different values of $\theta$ given observationally-equivalent consumption-savings decisions. Note that when calculating the welfare losses in the RS model, the value of $\beta$ is determined by (4.1) given the values of $\alpha$ and $\theta$, while the value of $\beta$ used in the basic RI model is just $1 / R$. Risk-

\footnotetext{
${ }^{24}$ This number varies largely for different individuals, from 2 to 20.5 is the average wealth/income ratio in the Survey of Consumer Finances 2001.
} 
sensitive consumers also suffer much more from finite information-processing constraints than do the consumers who are simply more patient.

What is the significance of these calculations? The fact that RS agents suffer much more from limited information processing capacity suggests that a careful experiment might be able to distinguish the two types of agents. If a researcher were to ask individuals 'What would you pay to receive more/better information about the state of the economy?' RS and RB agents would provide significantly different answers. Given the answers to this question, along with consumption-savings data, we may be able to determine whether agents are risk-sensitive or concerned about model misspecification, provided they in fact have limited informationprocessing capacity.

\section{Conclusion}

This paper provides a characterization of the consumption-savings behavior of a single agent who is both risk-sensitive and limited in their capacity to process signals. The key component to our model is the presence of precautionary savings, permitting the limited processing capacity to affect the average level of consumption. We show that there still exists an observational equivalence between models with and without risk-sensitivity even when agents face information-processing constraints, extending the results of Hansen, Sargent, and Tallarini (1999) to a broader class of models. However, within the observationally-equivalent class of models the welfare costs of rational inattention are not constant - models with higher discount factors and correspondingly lower risk-sensitivity generate larger welfare costs of RI than observationally-equivalent ones with lower discount factors and higher risk-sensitivity. We also show a connection between robustness, risk-sensitivity, and RI, in the sense that any combination of the three can plausibly generate the same consumption-savings decisions. However, as in the case with RS and RI, the welfare costs of RI are not equivalent across these observably-equivalent specifications.

While our model does permit precautionary savings, it is not as general as we would prefer. As we have noted above, solving fully-nonlinear versions of the RI model is extremely difficult given the shape of the optimal distribution of consumption, meaning that only models with short horizons or very small state spaces can be computed. ${ }^{25}$ We suspect that methods to deal with this problem can be developed, particularly for simple one-agent problems like the one studied here. A more difficult problem with RI models is noted in Sims (2005) - a general equilibrium setting will involve nonstandard features including inventories, retailing, and probably search. Implementation of RI solutions is also problematic - under general settings consumption is not a

\footnotetext{
${ }^{25}$ Sims (2006), Lewis (2006), Batchuluun, Luo, and Young (2007), and Tutino (2007) present some nonlinear solutions to RI models. These models are generally very small, either in terms of the number of states or the number of periods.
} 
deterministic function, meaning that the theory does not make clear predictions about what any individual's consumption will be, only what will prevail in a dataset of sufficiently large size. Despite these difficulties, we see rational inattention as an important tool for understanding consumer behavior.

\section{Appendix}

\subsection{Solving the RI PIH model}

To solve the standard PIH model, we first conjecture that the value function takes the following quadratic form

$$
\widehat{v}\left(\widehat{s}_{t}\right)=-A \widehat{s}_{t}^{2}-B \widehat{s}_{t}-C
$$

where $A_{0}, A_{1}$, and $A_{2}$ are constants to be determined. ${ }^{26}$ Substituting (7.1) into the Bellman equation,

$$
\widehat{v}\left(\widehat{s}_{t}\right)=\max _{c_{t}}\left\{-\frac{1}{2}\left(\bar{c}-c_{t}\right)^{2}+\beta E_{t}\left[\widehat{v}\left(\widehat{s}_{t+1}\right)\right]\right\}
$$

subject to the budget constraint, (2.6), and the Kalman filter equation, (3.6), yields

$$
-A \widehat{s}_{t}^{2}-B \widehat{s}_{t}-C=\max _{c_{t}}\left\{-\frac{1}{2}\left(\bar{c}-c_{t}\right)^{2}+\beta E_{t}\left[-A \widehat{s}_{t+1}^{2}-B \widehat{s}_{t+1}-C\right]\right\} .
$$

Second, performing the indicated optimization yields the following first-order condition

$$
-\left(c_{t}-\bar{c}\right)+\beta R B+2 \beta R^{2} A E_{t}\left[\widehat{s}_{t}-c_{t}\right]=0,
$$

so

$$
c_{t}=\frac{2 \beta R^{2} A}{1+2 \beta R^{2} A} \widehat{s}_{t}+\frac{\bar{c}+\beta R B}{1+2 \beta R^{2} A} .
$$

Substituting Equations (2.6), (3.6), and (7.4), into Equation (7.2) gives

$$
\begin{aligned}
& -A \widehat{s}_{t}^{2}-B \widehat{s}_{t}-C \\
& =-\frac{1}{2}\left(\frac{2 \beta R^{2} A}{1+2 \beta R^{2} A} \widehat{s}_{t}+\frac{\bar{c}+\beta R B}{1+2 \beta R^{2} A}-\bar{c}\right)^{2} \\
& -\beta C-\beta R B E_{t}\left[\frac{1}{1+2 \beta R^{2} A} \widehat{s}_{t}-\frac{\bar{c}+\beta R B}{1+2 \beta R^{2} A}\right] \\
& -\beta A\left\{\left(\frac{R}{1+2 \beta R^{2} A}\right)^{2} \widehat{s}_{t}^{2}-\frac{2 R}{1+2 \beta R^{2} A}\left(\frac{R(\bar{c}+\beta R B)}{1+2 \beta R^{2} A}\right) \widehat{s}_{t}+\operatorname{var}\left[\eta_{t+1}\right]+R^{2}\left(\frac{\bar{c}+\beta R B}{1+2 \beta R^{2} A}\right)^{2}\right\},
\end{aligned}
$$

\footnotetext{
${ }^{26}$ Luo (2007) solves the RI version of the PIH model with the $\beta R=1$ restriction.
} 
where $\eta_{t+1}=\theta\left[\left(\frac{\zeta_{t+1}}{1-(1-\theta) R L}\right)+\left(\xi_{t+1}-\frac{\theta R \xi_{t}}{1-(1-\theta) R L}\right)\right]$.

Third, collecting and matching terms, the constant coefficients turn out to be

$$
\begin{aligned}
& A=\frac{\beta R^{2}-1}{2 \beta R^{2}}, \\
& B=\frac{1-\beta R^{2}}{\beta R^{2}-\beta R} \bar{c}, \\
& C=\frac{\beta R^{2}-1}{2 \beta(R-1)^{2}} \bar{c}^{2}+\frac{\beta R^{2}-1}{2(1-\beta) R^{2}} \operatorname{var}\left[\eta_{t+1}\right],
\end{aligned}
$$

Substituting (7.5) and (7.6) into (7.4) yields

$$
c_{t}=\left(1-\frac{1}{\beta R^{2}}\right) \widehat{s}_{t}-\frac{1}{R-1}\left(1-\frac{1}{\beta R}\right) \bar{c}
$$

\subsection{Solving the RS model}

To solve the Bellman equation (2.14), we conjecture that

$$
v\left(s_{t}\right)=-A s_{t}^{2}-B s_{t}-C,
$$

where $A, B$, and $C$ are undetermined coefficients. We can then evaluate $E_{t}\left[\exp \left(-\alpha v\left(s_{t+1}\right)\right)\right]$ to obtain

$$
\begin{aligned}
& E_{t}\left[\exp \left(-\alpha v\left(s_{t+1}\right)\right)\right] \\
& =E_{t}\left[\exp \left(\alpha A s_{t+1}^{2}+\alpha B s_{t+1}+\alpha C\right)\right] \\
& =E_{t}\left[\exp \left(\alpha A R^{2}\left(s_{t}-c_{t}\right)^{2}+\alpha B R\left(s_{t}-c_{t}\right)+\left[2 \alpha A R\left(s_{t}-c_{t}\right)+\alpha B\right] \zeta_{t+1}+\alpha A \zeta_{t+1}^{2}+\alpha C\right)\right] \\
& =(1-2 c)^{-1 / 2} \exp \left(a+\frac{b^{2}}{2(1-2 c)}\right)
\end{aligned}
$$

where

$$
\begin{aligned}
a & =\alpha A R^{2}\left(s_{t}-c_{t}\right)^{2}+\alpha B R\left(s_{t}-c_{t}\right)+\alpha C, \\
b & =\left[2 \alpha A R\left(s_{t}-c_{t}\right)+\alpha B\right] \omega_{\zeta}, \\
c & =\alpha A \omega_{\zeta}^{2} .
\end{aligned}
$$


Thus, the distorted expectations operator can be written as

$$
\begin{aligned}
& \mathcal{R}_{t}\left[v\left(s_{t+1}\right)\right] \\
& =-\frac{1}{\alpha}\left\{-\frac{1}{2} \log (1-2 c)+a+\frac{b^{2}}{2(1-2 c)}\right\} \\
& =\frac{1}{2 \alpha} \log \left(1-2 \alpha A \omega_{\zeta}^{2}\right)-\frac{1}{\alpha}\left\{\alpha A R^{2}\left(s_{t}-c_{t}\right)^{2}+\alpha B R\left(s_{t}-c_{t}\right)+\alpha C+\frac{\left[2 \alpha A R\left(s_{t}-c_{t}\right)+\alpha B\right]^{2} \omega_{\zeta}^{2}}{2\left(1-2 \alpha A \omega_{\zeta}^{2}\right)}\right\} \\
& =\frac{1}{2 \alpha} \log \left(1-2 \alpha A \omega_{\zeta}^{2}\right)-\frac{A R^{2}}{1-2 \alpha A \omega_{\zeta}^{2}}\left(s_{t}-c_{t}\right)^{2}-\frac{B R}{1-2 \alpha A \omega_{\zeta}^{2}}\left(s_{t}-c_{t}\right)-\left[C+\frac{\alpha B^{2} \omega_{\zeta}^{2}}{2\left(1-2 \alpha A \omega_{\zeta}^{2}\right)}\right] .
\end{aligned}
$$

Maximizing the RHS of (2.14) with respect to $c_{t}$ yields the first-order condition

$$
-\left(c_{t}-\bar{c}\right)+\frac{2 \beta A R^{2}}{1-2 \alpha A \omega_{\zeta}^{2}}\left(s_{t}-c_{t}\right)+\frac{B \beta R}{1-2 \alpha A \omega_{\zeta}^{2}}=0
$$

which means that

$$
c_{t}=\frac{2 A \beta R^{2}}{1-2 \alpha A \omega_{\zeta}^{2}+2 A \beta R^{2}} s_{t}+\frac{\bar{c}\left(1-2 \alpha A \omega_{\zeta}^{2}\right)+B \beta R}{1-2 \alpha A \omega_{\zeta}^{2}+2 A \beta R^{2}} .
$$

Substituting (7.14) and (7.9) into (2.14) gives

$$
\begin{aligned}
& -A s_{t}^{2}-B s_{t}-C \\
& =-\frac{1}{2}\left(\frac{2 A \beta R^{2}}{1-2 \alpha A \omega_{\zeta}^{2}+2 A \beta R^{2}} s_{t}+\frac{B \beta R-2 A \beta R^{2} \bar{c}}{1-2 \alpha A \omega_{\zeta}^{2}+2 A \beta R^{2}}\right)^{2}+ \\
& \beta\left[\begin{array}{c}
\frac{1}{2 \alpha} \log \left(1-2 \alpha A \omega_{\zeta}^{2}\right)-\frac{A R^{2}}{1-2 \alpha A \omega_{\zeta}^{2}}\left(\frac{1-2 \alpha A \omega_{\zeta}^{2}}{1-2 \alpha A \omega_{\zeta}^{2}+2 A \beta R^{2}} s_{t}-\frac{\bar{c}\left(1-2 \alpha A \omega_{\zeta}^{2}\right)+B \beta R}{1-2 \alpha A \omega_{\zeta}^{2}+2 A \beta R^{2}}\right)^{2} \\
-\frac{B R}{1-2 \alpha A \omega_{\zeta}^{2}}\left(\frac{1-2 \alpha A \omega_{\zeta}^{2}}{1-2 \alpha A \omega_{\zeta}^{2}+2 A \beta R^{2}} s_{t}-\frac{\bar{c}\left(1-2 \alpha A \omega_{\zeta}^{2}\right)+B \beta R}{1-2 \alpha A \omega_{\zeta}^{2}+2 A \beta R^{2}}\right)-\left(C+\frac{\alpha B^{2} \omega_{\zeta}^{2}}{2\left(1-2 \alpha A \omega_{\zeta}^{2}\right)}\right)
\end{array}\right] .
\end{aligned}
$$


Collecting and matching terms, the constant coefficients turn out to be

$$
\begin{aligned}
A & =\frac{\beta R^{2}-1}{2 \beta R^{2}-2 \alpha \omega_{\zeta}^{2}}, \\
B & =\frac{R\left(1-\beta R^{2}\right)}{\left(\beta R^{2}-\alpha \omega_{\zeta}^{2}\right)(R-1)} \bar{c}, \\
C & =\frac{1}{2} \frac{R^{2}\left(\beta R^{2}-1\right)}{(R-1)^{2}\left(\beta R^{2}-\alpha \omega_{\zeta}^{2}\right)} \bar{c}^{2}+\frac{\beta}{1-\beta} \frac{1}{2 \alpha} \log \left(1-2 \alpha A \omega_{\zeta}^{2}\right),
\end{aligned}
$$

Substituting (7.15) and (7.16) into (7.14) yields the consumption function (2.17) in the text.

\subsection{Solving the RI-RS PIH model}

To obtain the solution to the RI-RS model we simply need to replace $\omega_{\zeta}^{2}$ with $\omega_{\eta}^{2}$ (we omit the steps):

$$
\begin{aligned}
A & =\frac{\beta R^{2}-1}{2 \beta R^{2}-2 \alpha \omega_{\eta}^{2}} \\
B & =\frac{R\left(1-\beta R^{2}\right)}{\left(\beta R^{2}-\alpha \omega_{\eta}^{2}\right)(R-1)} \bar{c} \\
C & =\frac{1}{2} \frac{R^{2}\left(\beta R^{2}-1\right)}{(R-1)^{2}\left(\beta R^{2}-\alpha \omega_{\eta}^{2}\right)} \bar{c}^{2}-\frac{\beta}{1-\beta} \frac{1}{2 \alpha} \log \left(1-2 \alpha A \omega_{\eta}^{2}\right)
\end{aligned}
$$

and the consumption function is

$$
c_{t}=\frac{\beta R^{2}-1}{\beta R^{2}-\alpha \omega_{\eta}^{2}} \widehat{s}_{t}+\frac{\left(1-\alpha \omega_{\eta}^{2}\right)(R-1)+1-\beta R^{2}}{\left(\beta R^{2}-\alpha \omega_{\eta}^{2}\right)(R-1)} \bar{c}
$$

Using this consumption function, the evolution of permanent income is then

$$
\begin{aligned}
s_{t+1} & =R\left(s_{t}-c_{t}\right)+\zeta_{t+1} \\
& =R s_{t}-R \frac{\beta R^{2}-1}{\beta R^{2}-\alpha \omega_{\eta}^{2}} \widehat{s}_{t}-R \frac{\left(1-\alpha \omega_{\eta}^{2}\right)(R-1)+1-\beta R^{2}}{\left(\beta R^{2}-\alpha \omega_{\eta}^{2}\right)(R-1)} \bar{c}+\zeta_{t+1},
\end{aligned}
$$

while perceived permanent income evolves according to

$$
\begin{aligned}
\widehat{s}_{t+1} & =(1-\theta) R\left(\widehat{s}_{t}-c_{t}\right)+\theta\left(s_{t+1}+\xi_{t+1}\right) \\
& =\frac{(1-\theta) R\left(1-\alpha \omega_{\eta}^{2}\right)-\theta R\left(\beta R^{2}-1\right)}{\beta R^{2}-\alpha \omega_{\eta}^{2}} \widehat{s}_{t}+\theta R s_{t}-R \frac{\left(1-\alpha \omega_{\eta}^{2}\right)(R-1)+1-\beta R^{2}}{\left(\beta R^{2}-\alpha \omega_{\eta}^{2}\right)(R-1)} \bar{c}+\theta\left(\zeta_{t+1}+\xi_{t+1}\right) .
\end{aligned}
$$




\subsection{Solving the Robust RI Model}

As before, we conjecture that

$$
\widehat{v}\left(\widehat{s}_{t}\right)=-A \widehat{s}_{t}^{2}-B \widehat{s}_{t}-C
$$

where $A, B$, and $C$ are undetermined coefficients. Substituting this guessed value function, (3.21), and (3.22) into the Bellman equation gives

$$
-A \widehat{s}_{t}^{2}-B \widehat{s}_{t}-C=\max _{c_{t}} \min _{\nu_{t}}\left\{-\frac{1}{2}\left(\bar{c}-c_{t}\right)^{2}+\beta E_{t}\left[\vartheta \nu_{t}^{2}-A \widehat{s}_{t+1}^{2}-B \widehat{s}_{t+1}-C\right]\right\} .
$$

We can do the min and max operations in any order, so we choose to do the minimization first. The first-order condition for $\nu_{t}$ is

$$
2 \vartheta \nu_{t}-2 A E_{t}\left[\omega_{\zeta} \nu_{t}+R\left(\widehat{s}_{t}-c_{t}\right)\right] \omega_{\zeta}-B \omega_{\zeta}=0
$$

which means that

$$
\nu_{t}=\frac{B+2 A R\left(\widehat{s}_{t}-c_{t}\right)}{2\left(\vartheta-A \omega_{\zeta}^{2}\right)} \omega_{\zeta}
$$

Substituting (7.21) back the Bellman equation (3.20) gives

$-A \widehat{s}_{t}^{2}-B \widehat{s}_{t}-C=\max _{c_{t}}\left\{-\frac{1}{2}\left(\bar{c}-c_{t}\right)^{2}+\beta E_{t}\left[\vartheta\left[\frac{B+2 A R\left(\widehat{s}_{t}-c_{t}\right)}{2\left(\vartheta-A \omega_{\zeta}^{2}\right)} \omega_{\zeta}\right]^{2}-A \widehat{s}_{t+1}^{2}-B \widehat{s}_{t+1}-C\right]\right\}$,

where

$$
\begin{aligned}
\widehat{s}_{t+1} & =(1-\theta)\left[R\left(\widehat{s}_{t}-c_{t}\right)+\omega_{\zeta} \nu_{t}\right]+\theta\left(R\left(s_{t}-c_{t}\right)+\omega_{\zeta} \nu_{t}+\zeta_{t+1}+\xi_{t+1}\right) \\
& =(1-\theta) R\left(\widehat{s}_{t}-c_{t}\right)+\theta R\left(s_{t}-c_{t}\right)+\omega_{\zeta} \nu_{t}+\theta\left(\zeta_{t+1}+\xi_{t+1}\right) \\
& =R\left(\widehat{s}_{t}-c_{t}\right)+\omega_{\zeta} \nu_{t}+\eta_{t+1}
\end{aligned}
$$

The first-order condition for $c_{t}$ is

$$
\left(\bar{c}-c_{t}\right)+2 \beta A\left(R+\frac{A R \omega_{\zeta}^{2}}{\vartheta-A \omega_{\zeta}^{2}}\right)\left[R\left(\widehat{s}_{t}-c_{t}\right)+\omega_{\zeta} \nu_{t}\right]+\beta B\left(R+\frac{A R \omega_{\zeta}^{2}}{\vartheta-A \omega_{\zeta}^{2}}\right)-2 \beta \vartheta \frac{A R \omega_{\zeta}}{\vartheta-A \omega_{\zeta}^{2}} \nu_{t}=0
$$

Using the solution for $\nu_{t}$ the solution for consumption is

$$
c_{t}=\frac{2 A \beta R^{2}}{1-A \omega_{\zeta}^{2} / \vartheta+2 A \beta R^{2}} \widehat{s}_{t}+\frac{\bar{c}\left(1-A \omega_{\zeta}^{2} / \vartheta\right)+B \beta R}{1-A \omega_{\zeta}^{2} / \vartheta+2 A \beta R^{2}}
$$


Substituting the above expressions into the Bellman equation gives

$$
\begin{aligned}
& -A \widehat{s}_{t}^{2}-B \widehat{s}_{t}-C \\
& =\left\{\begin{array}{c}
-\frac{1}{2}\left(\frac{2 A \beta R^{2}}{1-A \omega_{\zeta}^{2} / \vartheta+2 A \beta R^{2}} \widehat{s}_{t}+\frac{-2 A \beta R^{2} \bar{c}+B \beta R}{1-A \omega_{\zeta}^{2} / \vartheta+2 A \beta R^{2}}\right)^{2}+\beta \vartheta\left[\frac{1}{2} \frac{B-2 A R c}{2 A \beta \vartheta R^{2}-A \omega_{\zeta}^{2}+\vartheta}+\frac{A R}{\vartheta-A \omega_{\zeta}^{2}+2 A \beta R^{2} \vartheta} \widehat{s}_{t}\right]^{2} \omega_{\zeta}^{2} \\
+\beta E_{t}\left[\begin{array}{c}
-A\left\{\frac{R \vartheta}{2 A \beta \vartheta R^{2}-A \omega_{\zeta}^{2}+\vartheta} \widehat{s}_{t}-\frac{1}{2} \frac{2 B \beta \vartheta R^{2}+2 c \vartheta R-B \omega_{\zeta}^{2}}{2 A \beta \vartheta R^{2}-A \omega_{\zeta}^{2}+\vartheta}+\eta_{t+1}\right\}^{2} \\
-B\left\{\frac{R \vartheta}{2 A \beta \vartheta R^{2}-A \omega_{\zeta}^{2}+\vartheta} \widehat{s}_{t}-\frac{1}{2} \frac{2 B \beta \vartheta R^{2}+2 c \vartheta R-B \omega_{\zeta}^{2}}{2 A \beta \vartheta R^{2}-A \omega_{\zeta}^{2}+\vartheta}+\eta_{t+1}\right\}-C
\end{array}\right\}
\end{array}\right.
\end{aligned}
$$

Collecting and matching terms, the constant coefficients turn out to be

$$
\begin{aligned}
A & =\frac{\beta R^{2}-1}{2 \beta R^{2}-\omega_{\zeta}^{2} / \vartheta} \\
B & =\frac{R}{R-1} \frac{1-\beta R^{2}}{\beta R^{2}-\omega_{\zeta}^{2} /(2 \vartheta)} \bar{c} \\
C & =\frac{1}{2} \frac{R^{2}\left(\beta R^{2}-1\right)}{\left(\beta R^{2}-\omega_{\zeta}^{2} /(2 \vartheta)\right)(R-1)^{2}} \bar{c}^{2}+\frac{\beta}{1-\beta} \frac{\beta R^{2}-1}{2 \beta R^{2}-\omega_{\zeta}^{2} / \vartheta} \omega_{\eta}^{2}
\end{aligned}
$$

Substituting (7.25) and (7.26) into (7.23) yields the consumption function (3.25) in the text.

\subsection{Observational Equivalence Between RI-RS and RI-RB}

Comparing (3.9) with (3.25), we can derive the OE between RS and RB under RI:

$$
\alpha \omega_{\eta}^{2}=\omega_{\zeta}^{2} /(2 \vartheta)
$$

which implies that

$$
\alpha \frac{\theta}{1-(1-\theta) R^{2}}=\frac{1}{2 \vartheta} \text {. }
$$

Note that when $\beta R=1$, the standard RI PIH model implies that

$$
c_{t}=\frac{R-1}{R} \widehat{s}_{t} .
$$

Hence, under RS-RI $(\alpha>0)$, we can construct the following OE between $\alpha$ and $\beta$

$$
\frac{\beta R^{2}-1}{\beta R^{2}-\alpha \omega_{\eta}^{2}}=\frac{R-1}{R}
$$

which implies that

$$
\beta^{R I, R S}=\frac{R-(R-1) \alpha \omega_{\eta}^{2}}{R^{2}}<\frac{1}{R}
$$


Similarly, under RS-RI $(\vartheta>0)$, we can construct the following OE between $\beta$ and $\vartheta$

$$
\frac{\beta R^{2}-1}{\beta R^{2}-\omega_{\zeta}^{2} /(2 \vartheta)}=\frac{R-1}{R},
$$

which implies that

$$
\beta^{R I, R B}=\frac{R-(R-1) \omega_{\zeta}^{2} /(2 \vartheta)}{R^{2}}<\frac{1}{R} .
$$

Given the $O E$ under $R I$, the value function under RB-RI can be written as

$$
\begin{aligned}
\widehat{v}^{R B}\left(\widehat{s}_{t}\right) & =-\frac{\beta R^{2}-1}{2 \beta R^{2}-\omega_{\zeta}^{2} / \vartheta} \widehat{s}_{t}^{2}+\frac{\left(\beta R^{2}-1\right) R}{\left(\beta R^{2}-\omega_{\zeta}^{2} /(2 \vartheta)\right)(R-1)} \widehat{c}_{t} \\
& -\left(\frac{1}{2} \frac{R^{2}\left(\beta R^{2}-1\right)}{\left(R^{2} \beta-\omega_{\zeta}^{2} /(2 \vartheta)\right)(R-1)^{2}} \bar{c}^{2}+\frac{\beta}{1-\beta} \frac{\beta R^{2}-1}{2 \beta R^{2}-\omega_{\zeta}^{2} / \vartheta} \omega_{\eta}^{2}\right) \\
& =-\frac{R-1}{2 R} \widehat{s}_{t}^{2}+\bar{c} \widehat{s}_{t}-\left(\frac{1}{2} \frac{R}{R-1} \bar{c}^{2}+\frac{\beta}{1-\beta} \frac{R-1}{2 R} \omega_{\eta}^{2}\right),
\end{aligned}
$$

and the value function under RS-RI can be written as

$$
\begin{aligned}
\widehat{v}^{R S}\left(\widehat{s}_{t}\right) & =-\frac{\beta R^{2}-1}{2 \beta R^{2}-2 \alpha \omega_{\eta}^{2}} \widehat{s}_{t}^{2}+\frac{\left(\beta R^{2}-1\right) R}{\left(\beta R^{2}-\alpha \omega_{\eta}^{2}\right)(R-1)} \widehat{c}_{t} \\
& -\left(\frac{1}{2} \frac{R^{2}\left(\beta R^{2}-1\right)}{(R-1)^{2}\left(\beta R^{2}-\alpha \omega_{\eta}^{2}\right)} \bar{c}^{2}-\frac{\beta}{1-\beta} \frac{1}{2 \alpha} \log \left(1-2 \alpha A \omega_{\eta}^{2}\right)\right) \\
& =-\frac{R-1}{2 R} \widehat{s}_{t}^{2}+\widehat{c}_{t}-\left(\frac{1}{2} \frac{R}{R-1} \bar{c}^{2}-\frac{\beta}{1-\beta} \frac{1}{2 \alpha} \log \left(1-2 \alpha A \omega_{\eta}^{2}\right)\right)
\end{aligned}
$$

Therefore, the only difference between the two value functions is the constant term. Note that

$$
\lim _{\alpha \rightarrow 0}\left[-\frac{1}{\alpha} \log \left(1-\frac{R-1}{R} \alpha \omega_{\eta}^{2}\right)\right]=\frac{R-1}{R} \omega_{\eta}^{2}
$$

and

$$
\begin{aligned}
\frac{\partial\left(-\frac{1}{2 \alpha} \log \left(1-\frac{R-1}{R} \alpha \omega_{\eta}^{2}\right)\right)}{\partial \alpha} & =-\frac{1}{2 \alpha^{2}}\left(\frac{-(R-1) \alpha \omega_{\eta}^{2}}{R-(R-1) \alpha \omega_{\eta}^{2}}-\log \left(1-\frac{R-1}{R} \alpha \omega_{\eta}^{2}\right)\right) \\
& \simeq-\frac{1}{2 \alpha^{2}}\left(\frac{-(R-1) \alpha \omega_{\eta}^{2}}{R-(R-1) \alpha \omega_{\eta}^{2}}+\frac{(R-1) \alpha \omega_{\eta}^{2}}{R}\right) \\
& =\frac{1}{2 \alpha^{2}} \frac{\left[(R-1) \alpha \omega_{\eta}^{2}\right]^{2}}{R\left[R-(R-1) \alpha \omega_{\eta}^{2}\right]}>0 ;
\end{aligned}
$$


the second line follows whenever $\frac{R-1}{R} \alpha \omega_{\eta}^{2}$ is a small number. Hence,

$$
-\frac{1}{2 \alpha} \log \left(1-\frac{R-1}{R} \alpha \omega_{\eta}^{2}\right)>\frac{R-1}{2 R} \omega_{\eta}^{2} \text { for } \alpha>0
$$

that is, RS consumers suffer larger welfare losses from RI than do RB consumers. 


\section{References}

[1] Batchuluun, Altantsetseg, Yulei Luo, and Eric R. Young (2007), "Portfolio Choice with Information-Processing Limits," mimeo, University of Virginia and Hong Kong University.

[2] Cochrane, John H. (1989), "The Sensitivity of Tests of the Intertemporal Allocation of Consumption to Near-Rational Alternatives," American Economic Review 79(3), pp. 31937.

[3] Cover, Thomas M. and Joy A. Thomas (1991), Elements of Information Theory, John Wiley and Sons.

[4] Epstein, Lawrence G. and Stanley E. Zin (1989), "Substitution, Risk Aversion, and the Temporal Behavior of Consumption and Asset Returns: A Theoretical Framework," Econometrica 57, pp. 937-69.

[5] Flavin, Marjorie A. (1981), "The Adjustment of Consumption to Changing Expectations About Future Income," Journal of Political Economy 89, pp. 974-1009.

[6] Friedman, Milton (1957), A Theory of the Consumption Function, National Bureau of Economic Research.

[7] Hall, Robert E. (1978), "Stochastic Implications of the Life Cycle-Permanent Income Hypothesis: Theory and Evidence," Journal of Political Economy 86(6), pp. 971-87.

[8] Hansen, Lars Peter and Thomas J. Sargent (1995), "Discounted Linear Exponential Quadratic Gaussian Control," IEEE Transactions on Automatic Control 40, pp. 968-71.

[9] Hansen, Lars Peter and Thomas J. Sargent (2004), "'Certainty Equivalence' and 'Model Uncertainty,"' working paper, University of Chicago and New York University.

[10] Hansen, Lars Peter and Thomas J. Sargent (2007), Robustness, forthcoming, Princeton University Press.

[11] Hansen, Lars Peter, Thomas J. Sargent, and Thomas D. Tallarini, Jr. (1999), "Robust Permanent Income and Pricing," Review of Economic Studies 66, pp. 873-907.

[12] Jacobson, David H. (1973), "Optimal Stochastic Linear Systems with Exponential Performance Criteria and Their Relation to Deterministic Differential Games," IEEE Transactions on Automatic Control 18, pp. 124-31.

[13] Kasa, Kenneth (2006), "Robustness and Information Processing," Review of Economic Dynamics 9(1), pp. 1-33. 
[14] Kimball, Miles S. (1990), "Precautionary Savings in the Small and the Large," Econometrica 58(1), pp. 53-73.

[15] Kreps, David M. and Evan L. Porteus (1978), "Temporal Resolution of Uncertainty and Dynamic Choice Theory," Econometrica 46, pp. 185-200.

[16] Lewis, Kurt F. (2006), "The Life-Cycle Effects of Information-Processing Constraints," mimeo, University of Iowa.

[17] Luo, Yulei (2007), "Consumption Dynamics Under Information Processing Constraints," forthcoming, Review of Economic Dynamics.

[18] Luo, Yulei and Eric R. Young (2007), "Rational Inattention and Aggregate Fluctuations," mimeo, Hong Kong University and University of Virginia.

[19] Pischke, Jorn-Steffen (1995), "Individual Income, Incomplete Information, and Aggregate Consumption," Econometrica 63 (4), pp. 805-40.

[20] Reis, Ricardo (2006), "Inattentive Consumers," Journal of Monetary Economics 53(8), pp. $1761-800$.

[21] Shannon, Claude (1948), "A Mathematical Theory of Communication," The Bell System Technical Journal 27, pp. 623-56.

[22] Simon, Herbert A. (1956), "Dynamic Programming Under Uncertainty with a Quadratic Criterion Function," Econometrica 24(1), pp. 74-81.

[23] Sims, Christopher A. (1998), "Stickiness," Carnegie-Rochester Conference Series on Public Policy 49, pp. 317-56.

[24] Sims, Christopher A. (2003), "Implications of Rational Inattention," Journal of Monetary Economics 50(3), pp. 665-90.

[25] Sims, Christopher A. (2005), "Rational Inattention: A Research Agenda," Technical Report, Princeton University.

[26] Sims, Christopher A. (2006), "Rational Inattention: Beyond the Linear-Quadratic Case," American Economic Review 96(2), pp. 158-163.

[27] Tallarini, Thomas D., Jr. (2000), "Risk-Sensitive Real Business Cycles," Journal of Monetary Economics 45(3), pp. 507-32.

[28] Theil, Henri (1957), "A Note on Certainty Equivalence in Dynamic Planning," Econometrica 25(2), pp. 346-9. 
[29] Tutino, Antonella (2007), "The Rigidity of Choice: Life Cycle Savings with InformationProcessing Limits," mimeo, Princeton University.

[30] van der Ploeg, Frederick (1993), "A Closed-Form Solution for a Model of Precautionary Saving," Review of Economic Studies 60(2), pp. 385-95.

[31] Whittle, Peter (1981), "Risk-Sensitive Linear/Quadratic/Gaussian Control," Advances in Applied Probability 13, pp. 764-77.

[32] Whittle, Peter (1990), Risk-Sensitive Optimal Control, John Wiley and Sons. 


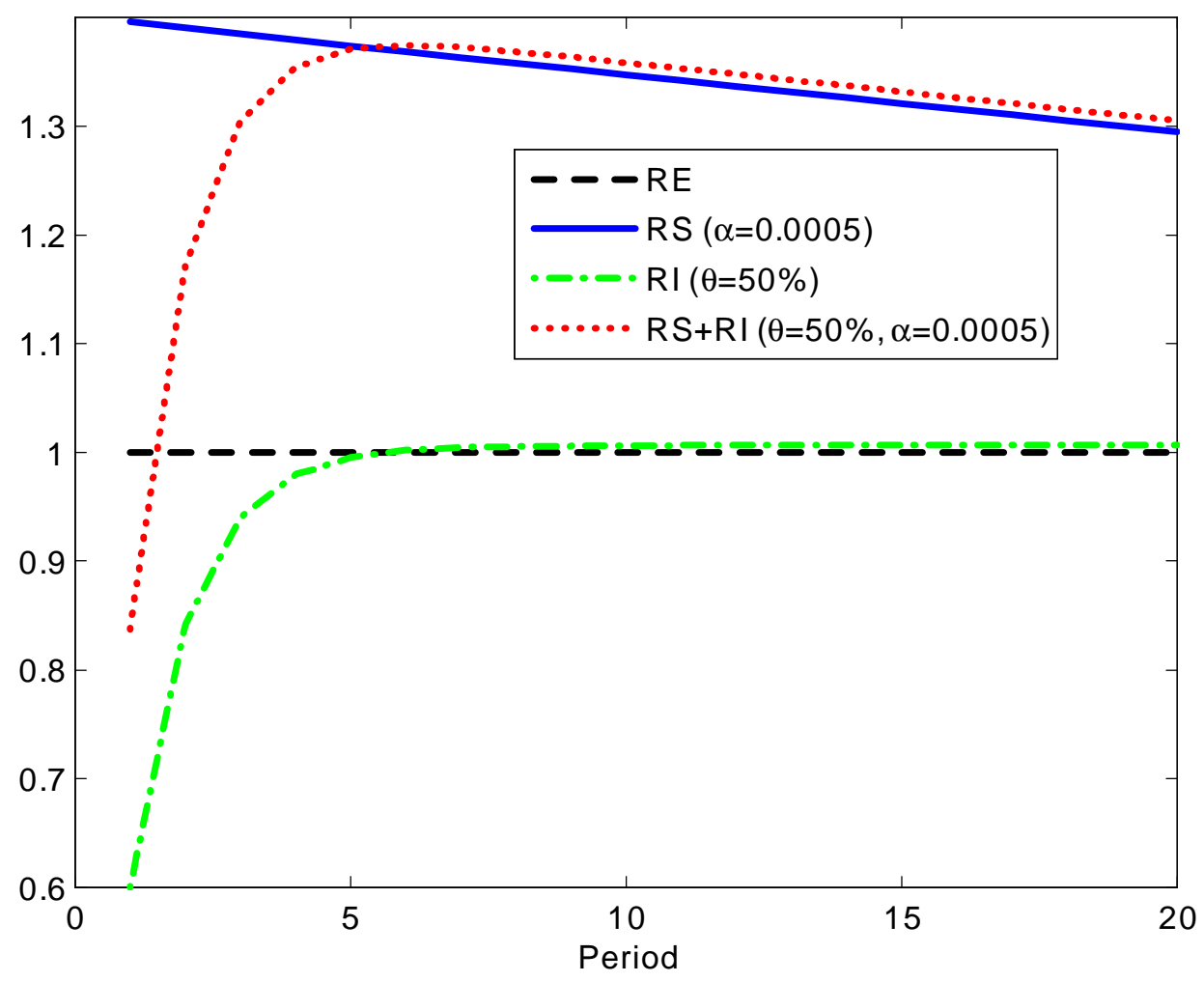

Figure 1: Responses of consumption to income shock, $\varepsilon$ 


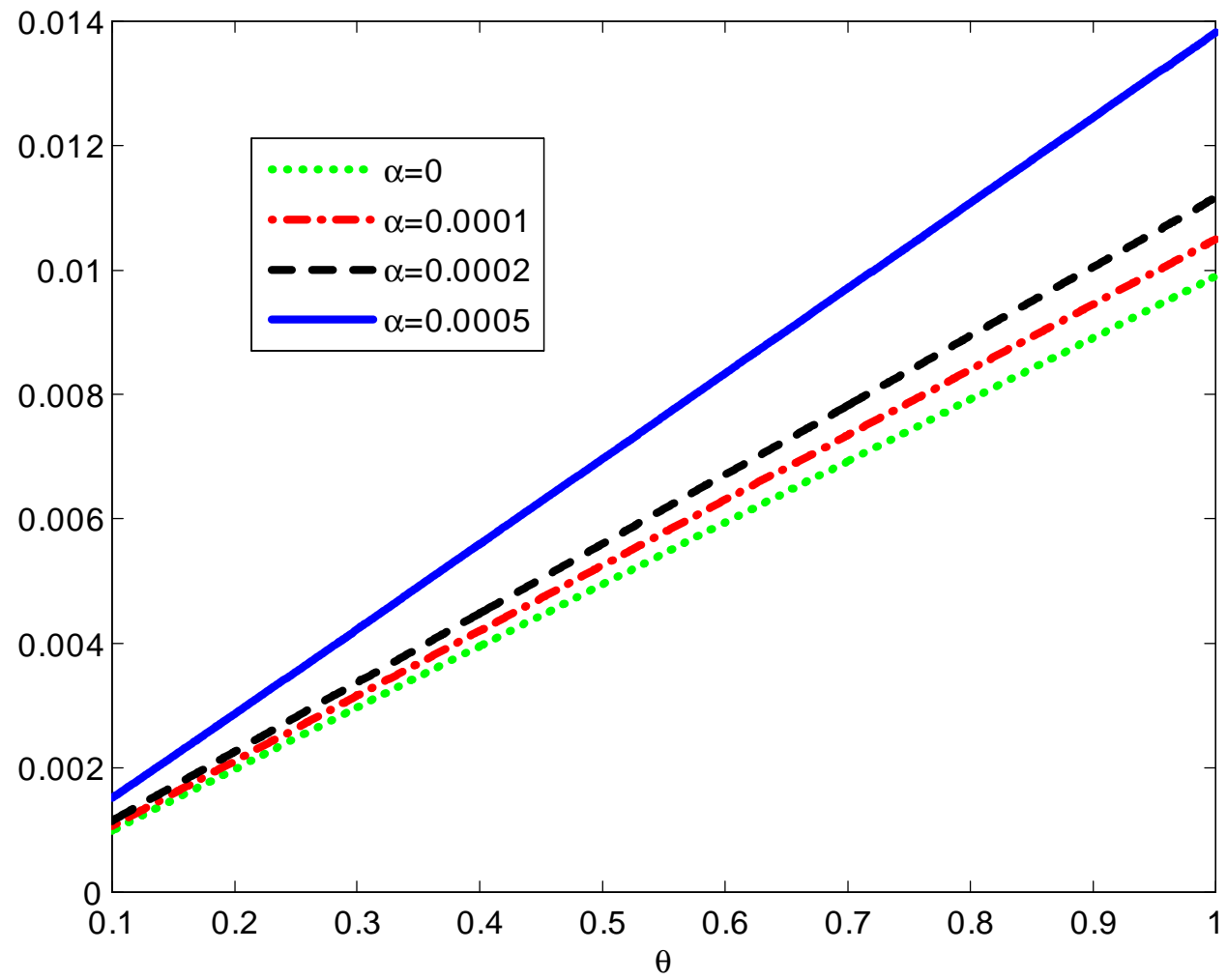

Figure 2: The MPC out of financial wealth $w$ and human wealth $\frac{y}{R-1}$ 


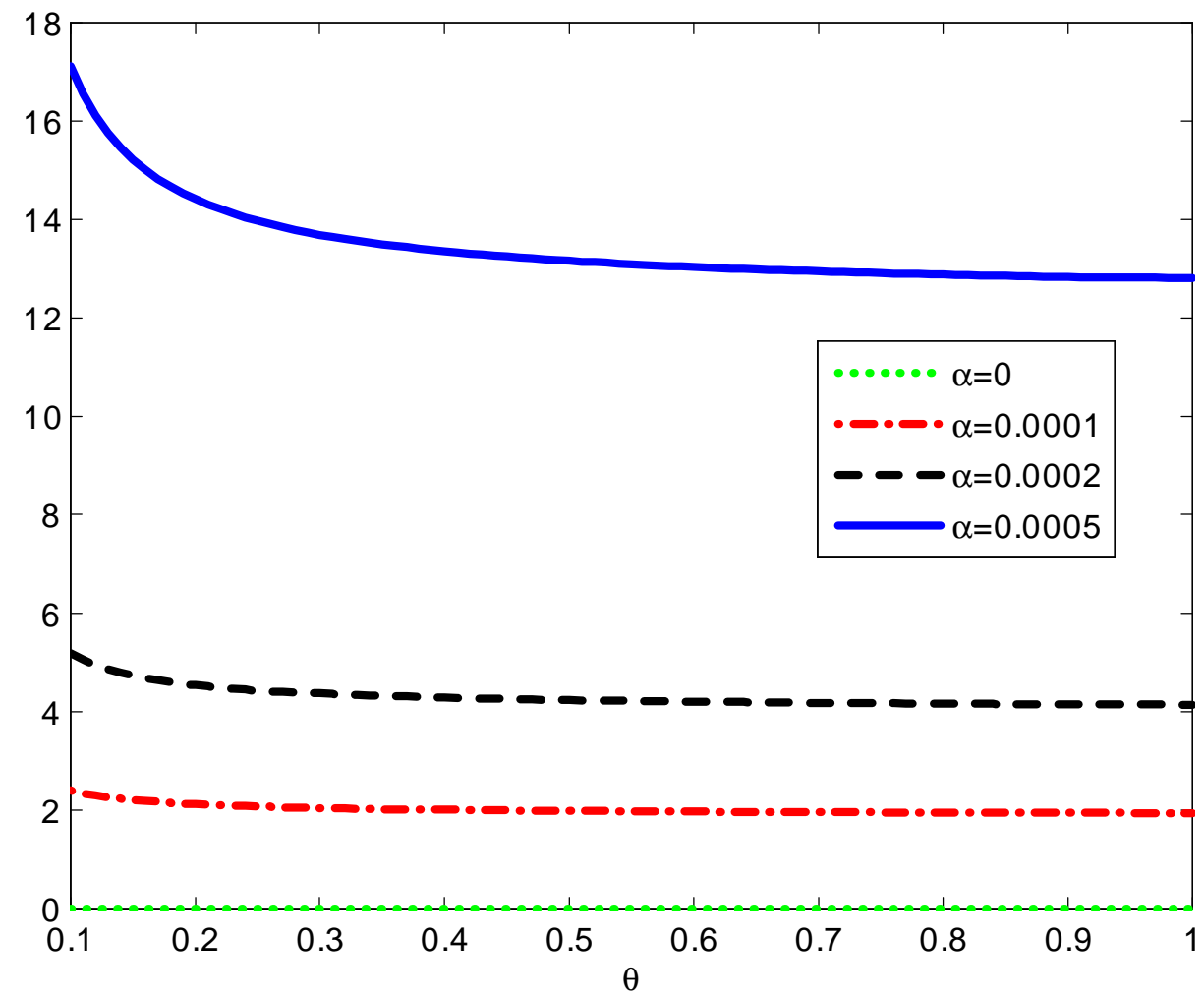

Figure 3: The effect of the combination of $(\alpha, \theta)$ on precautionary savings 


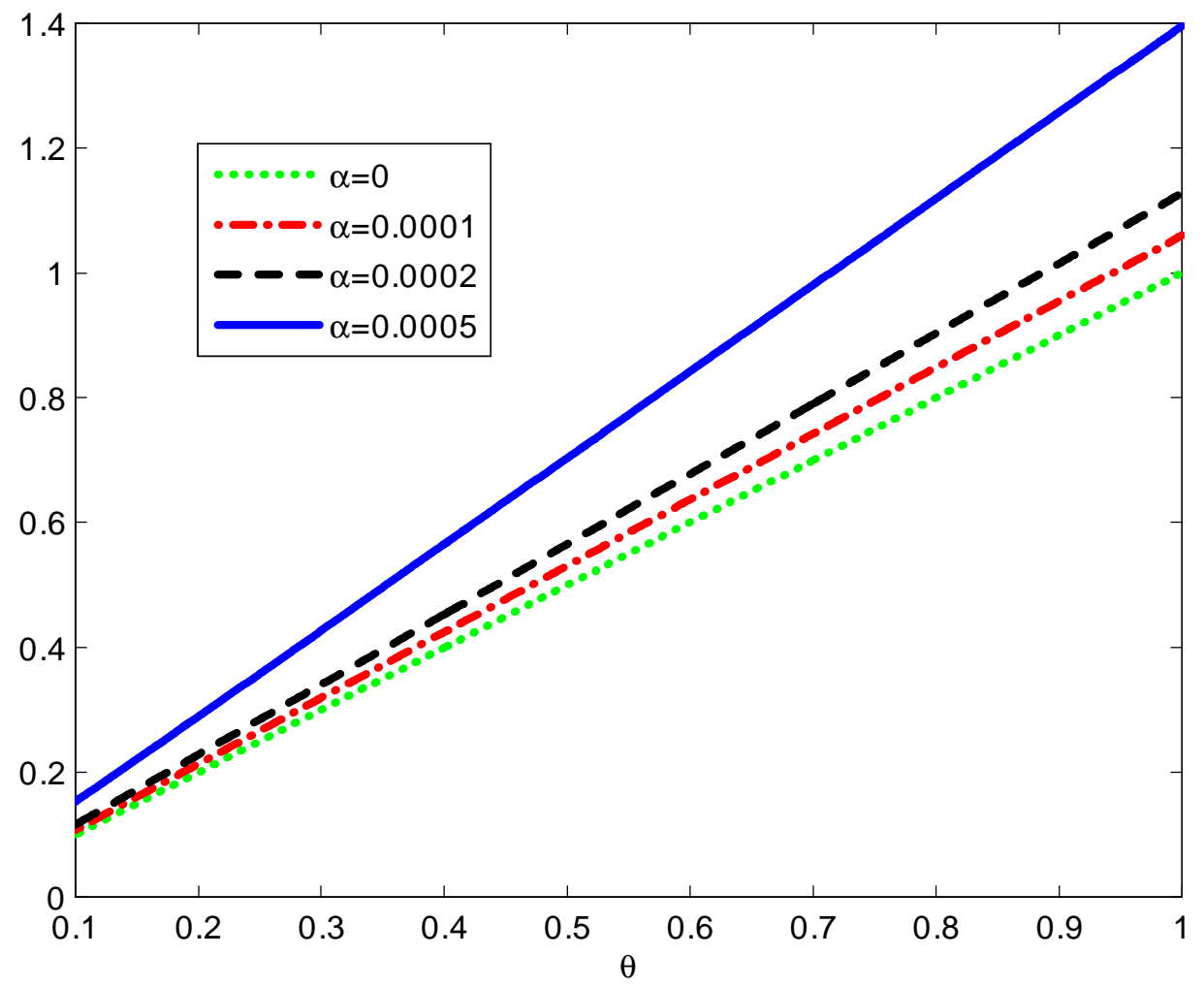

Figure 4: Short-run responses of consumption 


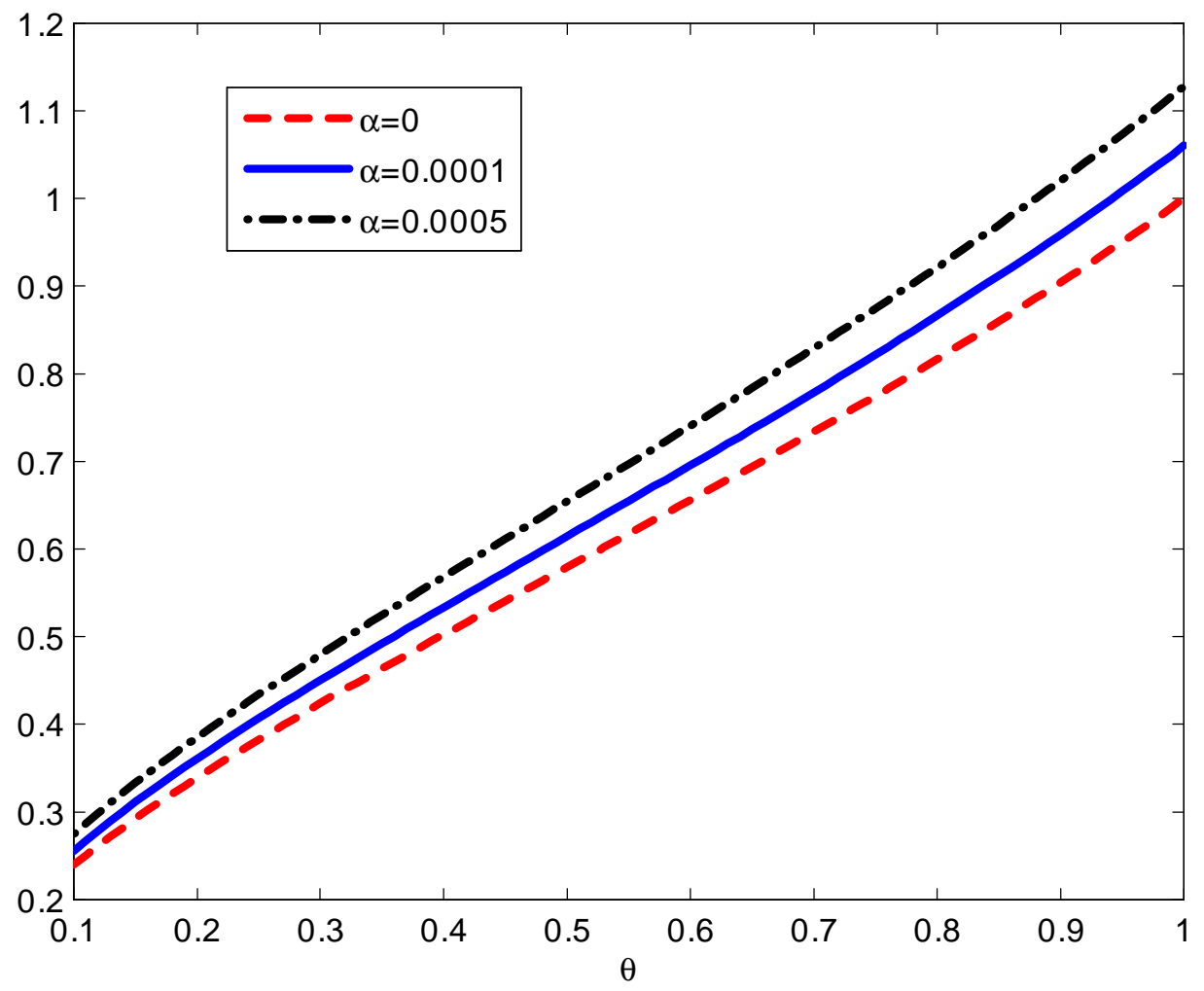

Figure 5: The smoothness of aggregate consumption in terms of $(\alpha, \theta)$ 


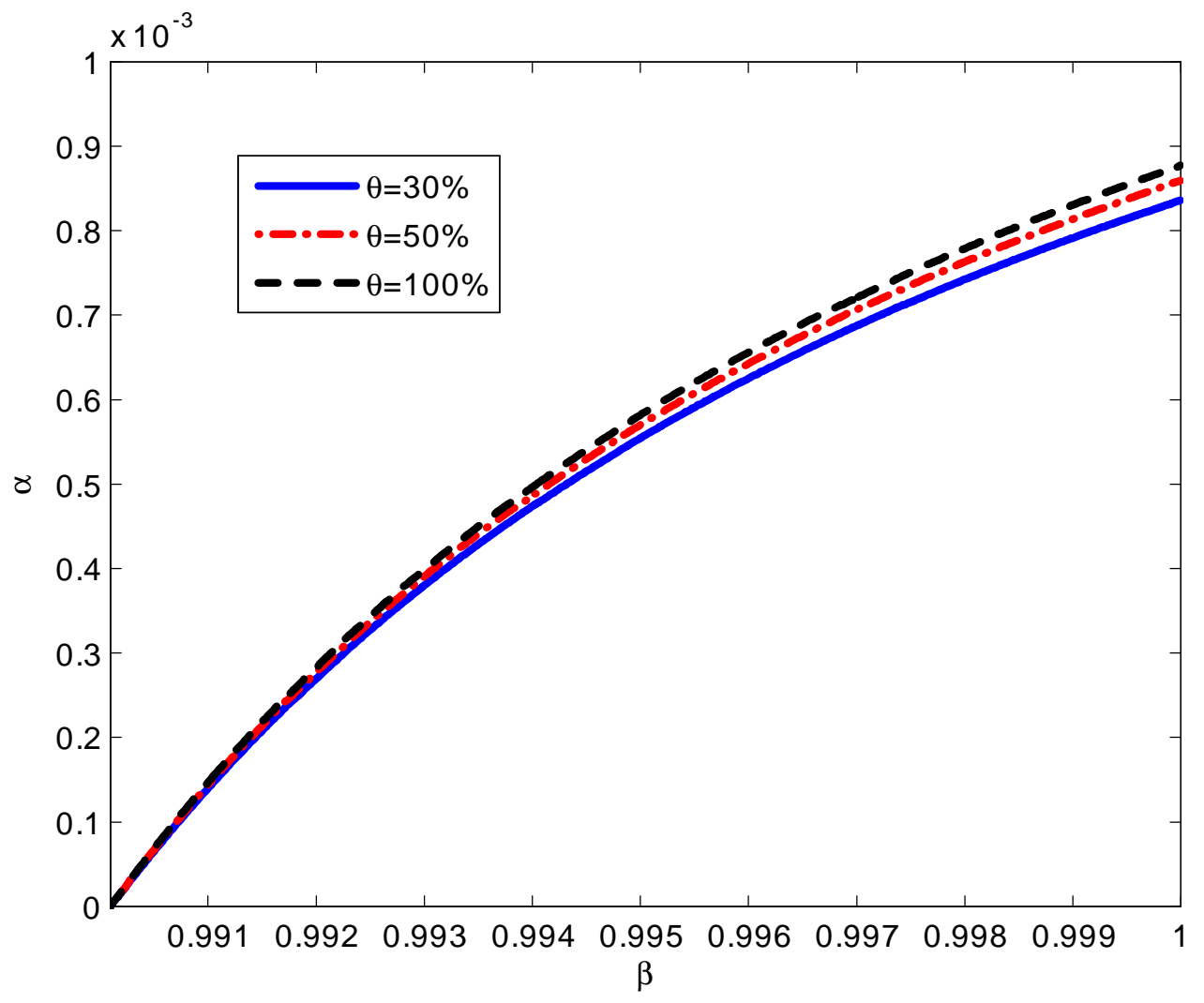

Figure 6: The observational equivalence between $\alpha$ and $\beta$ 
Table 1: Comparison of RI-induced welfare losses the RS-RI and RB-RI models

\begin{tabular}{|l|cccc|}
\hline \multicolumn{5}{|c|}{$\Delta^{R S} / \Delta^{R B}$} \\
\hline & $(\alpha, \vartheta)=\left(5 \cdot 10^{-6}, 10^{5}\right)$ & $\left(10^{-5}, 5 \cdot 10^{4}\right)$ & $\left(5 \cdot 10^{-5}, 10^{4}\right)$ & $\left(10^{-4}, 5 \cdot 10^{3}\right)$ \\
\hline$\theta=10 \%$ & 2.2473 & 2.5040 & 4.7313 & 7.9944 \\
$\theta=40 \%$ & 2.2460 & 2.5013 & 4.7050 & 7.9028 \\
$\theta=70 \%$ & 2.2461 & 2.5012 & 4.7023 & 7.8928 \\
$\theta=90 \%$ & 2.2459 & 2.5009 & 4.7010 & 7.8891 \\
\hline
\end{tabular}

Note. The parameters are set as follows: $\bar{c}=32, E[y]=16, R=1.01$, and $\omega_{\varepsilon}=0.2559$.

Table 2: Comparison of RI-induced welfare losses in the RS-RI and basic RI models

\begin{tabular}{|c|cccc|}
\hline \multicolumn{5}{|c|}{$\Delta^{R S} / \Delta^{\beta}$} \\
\hline & $(\alpha, \beta)=\left(5 \cdot 10^{-6}, 0.9901\right)$ & $\left(10^{-5}, 0.9901\right)$ & $\left(5 \cdot 10^{-5}, 0.9901\right)$ & $\left(10^{-4}, 0.9901\right)$ \\
\hline$\theta=10 \%$ & 2.2345 & 2.4757 & 4.4690 & 7.1318 \\
$\theta=40 \%$ & 2.2334 & 2.4730 & 4.4442 & 7.0501 \\
$\theta=70 \%$ & 2.2333 & 2.4728 & 4.4416 & 7.0411 \\
$\theta=90 \%$ & 2.2331 & 2.4726 & 4.4404 & 7.0379 \\
\hline
\end{tabular}

Note. The parameters are set as follows: $\bar{c}=32, E[y]=16, R=1.01$, and $\omega_{\varepsilon}=0.2559$. 